\title{
Nonlinear Dynamics of Cutting Process considering Higher-Order Deformation of Composite Cutting Tool
}

\author{
Donghui Yao (D), Yongsheng Ren (D), Yuhuan Zhang (D), and Bole Ma \\ College of Mechanical and Electronic Engineering, Shandong University of Science and Technology, Qingdao 266590, China \\ Correspondence should be addressed to Yongsheng Ren; renys@sdust.edu.cn
}

Received 8 September 2021; Revised 28 October 2021; Accepted 10 November 2021; Published 2 December 2021

Academic Editor: Marta Berardengo

Copyright ( $\odot 2021$ Donghui Yao et al. This is an open access article distributed under the Creative Commons Attribution License, which permits unrestricted use, distribution, and reproduction in any medium, provided the original work is properly cited.

\begin{abstract}
In this paper, the nonlinear dynamic analysis of the cutting process of composite cutting tool is performed. The cutting tool is simplified to a nonplanar bending rotating shaft. The higher-order bending deformation, structural damping, and gyroscopic effect of cutting tool are considered. It is assumed that cutting tool is subjected to a regenerative two-dimensional cutting force containing the first and second harmonic components. Based on the Hamilton principle, the motion equation of nonlinear chatter of the cutting system is derived. The nonlinear ordinary differential equations in the generalized coordinates are obtained by Galerkin method. In order to analyze the nonlinear dynamic response of cutting process, the multiscale method is used to derive the analytical approximate solution of the forced response for the cutting system under periodic cutting forces. In the forced response analysis, four cases including primary resonance and superharmonic resonance, i.e., $\bar{\Omega}=\omega_{1}, \bar{\Omega}=\omega_{2}, 2 \bar{\Omega}=\omega_{1}$, and $2 \bar{\Omega}=\omega_{2}$, are considered. The influences of ratio of length to diameter, structural damping, cutting force, and ply angle on primary resonance and superharmonic resonance are investigated. The results show that nonlinearity due to higher-order bending deformation significantly affects the dynamic behavior of the milling process and that the effective nonlinearity of the cutting system is of hard type. Multivalued resonance curves and jump phenomenon are presented. The influences of various factors, such as ratio of length to diameter, ply angle, structural damping, cutting force, and rotating speed, are thoroughly discussed.
\end{abstract}

\section{Introduction}

As a high-efficiency, high-quality, low-cost machining method, high-speed cutting technology has been widely used in aerospace and mold manufacturing. However, chatter reduces the cutting stability during machining operations, causes a decrease in the machining quality and cutting efficiency of the workpiece, damages the tool, and shortens the service life of the machine.

The passive chatter control methods are mainly based on various types of dynamic vibration absorbers $[1,2]$ and impact dampers [3]. However, composite materials are known to have higher static stiffness, damping, and specific stiffness. It has been found that the dynamic stiffness and fundamental natural frequency of cutter bar may be improved simultaneously if composite is employed [4-6]. This is very beneficial for the stability of high rotational speed machining for deep holes.
Within the framework of linear chatter theories and based on different theories, several of chatter phenomena as well as their stability boundary in different cutting processes were discovered [7-14]. Tobias [8] introduced the time-delay instability in the cutting force and proposed the regeneration theory, which is considered to provide a complete explanation of the chatter phenomenon so far.

However, because linear theory cannot predict some important dynamic behaviors of cutting process, nonlinear modeling of cutting systems has received more attention. The nonlinearity in cutting systems is mainly caused by structural nonlinearity, delayed nonlinearity of cutting force, etc. [15-18]. Hanna and Tobias [15] first proposed a timedelay nonlinear model with quadratic and cubic structural stiffness and cutting forces, which has inspired great interest in analyzing the global dynamics of the cutting system. The effective mathematical methods for the nonlinear theory of cutting systems include center manifold theory, bifurcation 
theory, perturbation analysis, phase portraits, and Poincaré sections.

Pratt [19] used the multiscale method, harmonic balance method, and Floquet theory to study the models of Hanna and Tobias [18]. The results showed that subcritical Hopf bifurcation may occur due to the existence of cubic structural nonlinearity. Moradi et al. [20] studied the existence of different types of bifurcation in the cutting process considering the tool wear and process damping. They developed a 2-dof linear model of the tool and a polynomial nonlinear model of the cutting force. In addition, the multiscale method was used to obtain the approximation analysis solution of primary resonance. Moradi et al. [21] used a model similar to that in literature [20] to analyze the forced vibration of the milling process. In the study, not only the primary resonance but also the superharmonic resonance and the internal resonance were investigated. Moradi et al. [22] investigated the internal resonance and regenerative chatter of the milling process considering both the cutting force and the structural nonlinearity.

However, in the above studies, the tool structure was simulated with 1-dof or 2-dof vibration system, in which the concentration parameters such as mass and stiffness were obtained through experiments or empirical method. There is no obvious correlation between these simplified models and the continuous system equations of the tool.

In order to investigate the dynamic characteristics of cutting system and the stability mechanism of machining process, it is necessary to conduct a comprehensive analysis on various influencing factors. In this case, if the empirical method is used to establish the model of the tool structure, a large number of repetitive tests are needed to obtain the dynamic parameters of the tool structure of different sizes, geometries, and materials, which is very time consuming and of low effectiveness. Therefore, as a more effective modeling method for cutting tool, the continuous parameter dynamics modeling of cutting tool based on analytical method, has received great attention [23-33].

However, in most of the existing continuous system dynamics models, the influence of the nonlinearity of the tool structure has not been considered. Therefore, the existing dynamic model is only suitable for analyzing the linear dynamics of the natural frequency, chatter frequency, dynamic deformation, and chatter stability of the cutting system. In addition, the current continuous system dynamic studies are mainly focused on the tool structure made of isotropic metal materials. Nevertheless, there is no research about the nonlinear dynamics of the process with anisotropy composite tool structures.
The structural nonlinearity is essentially arisen by the flexible nature of the cutting tools with small diameter or long extension part. The structural nonlinearity of the cutting tools may be described using higher-order large deformations [34] or geometric nonlinearity [35].

In this paper, the nonlinear dynamics of the cutting process with a composite cutting tool are investigated. The composite tool structure is simplified to a nonplanar cantilever rotating shaft. The structural nonlinearity is introduced by considering the higher-order deformation of cutting tool. It is assumed that the cutting tool is subjected to a regenerative cutting force containing harmonic components. Based on the Hamilton principle, the nonlinear chatter equation of the cutting system is derived. The Galerkin method is used to obtain the nonlinear ordinary differential equations in the generalized coordinates. The multiscale method is used to obtain the approximate solution of the forced vibration response of the cutting process subjected to periodic cutting forces. Nonlinear dynamics of the system are studied for four cases of primary and superharmonic resonances; i.e., $\bar{\Omega}=\omega_{1}, \bar{\Omega}=\omega_{2}, 2 \bar{\Omega}=\omega_{1}$, and $2 \bar{\Omega}=\omega_{2}$ are studied. The numerical calculation is conducted to investigate the effect of various parameters on the frequency response of the cutting system.

\section{Mathematical Model and Solution}

2.1. Kinetic Energy and Potential Energy. The structural sketch of composite cutting tool with nonplanar bending is shown in Figure 1. The kinetic energy of the rotating cutting tool without considering torsional deformation is as follows:

$$
T=\frac{1}{2} \int_{0}^{l}\left[\rho A\left(\dot{v}^{2}+\dot{w}^{2}\right)+\rho I\left(\dot{\psi}_{y}^{2}+\dot{\psi}_{z}^{2}\right)-4 \Omega \rho I \psi_{z} \dot{\psi}_{y}\right] d x,
$$

where $v$ and $w$ represent the displacement at any point on the neutral axis along the $y$ and $z$ directions, respectively. $\psi_{z}$ and $\psi_{y}$ represent bending rotation angles of the cross section around the $y$ and $z$ axes, respectively. $\rho A$ represents the mass per unit length, and $\rho I$ represents the mass moment of inertia of the cross section. The superposed dots represent derivatives with respect to the time $t$.

Using the expression of the displacement fields for Euler-Bernoulli beam and the stress-strain displacement relations of the composite cutting tool, as shown in Appendix $\mathrm{A}$, one can obtain the following expression for the potential energy of the rotating composite cutting tool with the higher-order deformation:

$$
U=\frac{1}{2} \int_{0}^{L} \iint_{A} \bar{Q}_{11}\left(z^{2}\left(\frac{\partial \psi_{y}}{\partial x}\right)^{2}+y^{2}\left(\frac{\partial \psi_{z}}{\partial x}\right)^{2}-2 y z \frac{\partial \psi_{y}}{\partial x} \frac{\partial \psi_{z}}{\partial x}+\frac{1}{4} \psi_{y}^{4}+\frac{1}{4} \psi_{z}^{4}+\frac{1}{2} \psi_{y}^{2} \psi_{z}^{2}-2\left(z \frac{\partial \psi_{y}}{\partial x}+y \frac{\partial \psi_{z}}{\partial x}\right)\left(\frac{1}{2} \psi_{y}^{2}+\frac{1}{2} \psi_{z}^{2}\right)\right)^{2} d A d x
$$




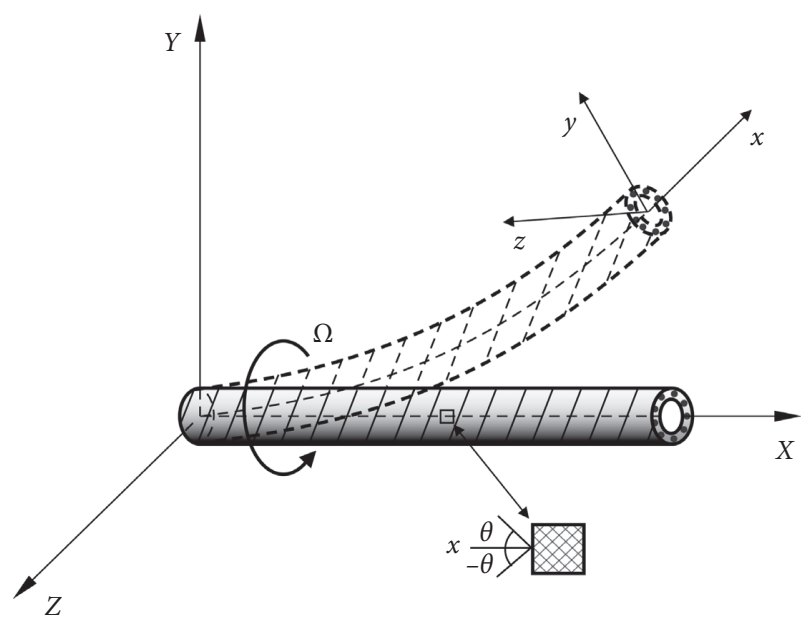

FIGURE 1: Composite cutting tool with nonplanar bending.

where $\bar{Q}_{11}$ refers to the off-axis stiffness coefficient of the $k$ th layer for the composite cutting tool.

Regardless of the influence of shear deformation, based on the Euler-Bernoulli beam theory, the bending rotation angles and the displacements have the following relationship:

$$
\psi_{z}=-\frac{\partial w}{\partial x}, \psi_{y}=\frac{\partial v}{\partial x}
$$

2.2. Damping Dissipative Energy Function and Virtual Work of Cutting Force. Rayleigh dissipative energy function of the cutting tool can be expressed as follows:

$$
W_{c}=\frac{1}{2} \int_{0}^{L} c\left(\dot{v}^{2}+\dot{w}^{2}\right) d x
$$

where $c$ is structural damping coefficient.

The virtual work of the cutting force $\delta W$ can be expressed as

$$
\delta W=\int_{0}^{L}\left(L_{v} \delta v+L_{w} \delta w\right) d x
$$

where $L_{v}=F_{y} \delta_{D}(x-L), L_{w}=F_{z} \delta_{D}(x-L)$, and $\delta_{D}(x-L)$ is the delta function.

The linear regenerative cutting forces $F_{y}$ and $F_{z}$ can be obtained by [20]

$$
\begin{aligned}
& F_{y}=-\frac{N}{2 \pi}\left[\alpha_{0}\left(v-v_{\tau}\right)+\beta_{0}\left(w-w_{\tau}\right)+\gamma_{0}\right]+\frac{c_{f}}{2}\left[\varsigma_{1} \cos (2 \Omega t-\pi / 2)-\eta_{1} \cos 2 \Omega t+\eta_{1}\right]+\left[\eta_{2} \cos (\Omega t-\pi / 2)+\varsigma_{2} \cos \Omega t\right] \\
& F_{z}=\frac{N}{2 \pi}\left[\alpha_{0}^{\prime}\left(v-v_{\tau}\right)+\beta_{0}^{\prime}\left(w-w_{\tau}\right)+\gamma_{0}^{\prime}\right]+\frac{c_{f}}{2}\left[\eta_{1} \cos (2 \Omega t-\pi / 2)+\varsigma_{1} \cos 2 \Omega t-\varsigma_{1}\right]+\left[\varsigma_{2} \cos (\Omega t-\pi / 2)-\eta_{2} \cos \Omega t\right],
\end{aligned}
$$

where

$$
\begin{aligned}
\alpha_{0} & =0.5 \varsigma_{1}+0.25 \pi \eta_{1}, \beta_{0}=0.5 \eta_{1}+0.25 \pi \varsigma_{1}, \\
\alpha_{0}^{\prime} & =-0.5 \eta_{1}+0.25 \pi \varsigma_{1}, \beta_{0}^{\prime}=0.5 \varsigma_{1}-0.25 \pi \eta_{1}, \\
\gamma_{0} & =\eta_{2}+\varsigma_{2}, \gamma_{0}^{\prime}=\varsigma_{2}-\eta_{2}, \\
\varsigma_{1} & =K_{t c} a, \varsigma_{2}=K_{t e} a, \\
\eta_{1} & =K_{r c} a, \eta_{2}=K_{r e} a, \\
v & =v(x, t), v_{\tau}=v(x, t-\tau), w=w(x, t), w_{\tau}=w(x, t-\tau),
\end{aligned}
$$

in which $\tau=2 \pi / N \Omega$ is the delay time of milling process; $N$ is the number of teeth; $\left(K_{\mathrm{tc}}, K_{\mathrm{te}}\right)$ and $\left(K_{\mathrm{rc}}, K_{\mathrm{re}}\right)$ are the cutting force coefficients in the tangential and radial directions, respectively; $a$ is the axial cutting depth; and $c_{f}$ is the feed per tooth per revolution.

2.3. Dynamic Model of Milling Process. In order to obtain the equation of motion of the cutting system, the principle of Hamilton is used as follows:

$$
\int_{t_{1}}^{t_{2}}\left(\delta U+\delta W_{c}-\delta T-\delta W\right) d t=0 .
$$

Substituting (1), (2), (4), and (5) into (8), the equations of motion in both $y$ and $z$ directions can be obtained:

$$
\begin{aligned}
& (m)_{\text {equiv }} \ddot{v}+c \dot{v}-2(\rho I)_{\text {equiv }} \Omega \dot{w}^{\prime \prime}-(\rho I)_{\text {equiv }} \ddot{v}^{\prime \prime}+(E I)_{\text {equiv }} \frac{\partial^{4} v}{\partial x^{4}}+\frac{(E A)_{\text {equiv }}}{2}\left[\left(v^{\prime^{2}}+w^{\prime^{2}}\right) v^{\prime}\right]^{\prime}=F_{y} \delta_{D}(x-L), \\
& (m)_{\text {equiv }} \ddot{w}+c \dot{w}+2(\rho I)_{\text {equiv }} \Omega \dot{v}^{\prime \prime}-(\rho I)_{\text {equiv }} \ddot{w}^{\prime \prime}+(E I)_{\text {equiv }} \frac{\partial^{4} w}{\partial x^{4}}+\frac{(E A)_{\text {equiv }}}{2}\left[\left(v^{\prime^{2}}+w^{\prime^{2}}\right) w^{\prime}\right]^{\prime}=F_{z} \delta_{D}(x-L)
\end{aligned}
$$

where 


$$
\begin{aligned}
& (m)_{\text {equiv }}=(\rho A)_{\text {equiv }}=\pi \sum_{k=1}^{N} \rho_{k}\left(r_{k+1}^{2}-r_{k}^{2}\right), \\
& (\rho I)_{\text {equiv }}=\frac{\pi}{4} \sum_{k=1}^{N} \rho_{k}\left(r_{k+1}^{4}-r_{k}^{4}\right) \\
& (E I)_{\text {equiv }}=\frac{\pi}{4} \sum_{k=1}^{N} \bar{Q}_{11}\left(r_{k+1}^{4}-r_{k}^{4}\right) \\
& (E A)_{\text {equiv }}=\pi \sum_{k=1}^{N} \bar{Q}_{11}\left(r_{k+1}^{2}-r_{k}^{2}\right) .
\end{aligned}
$$

Here, $(E I)_{\text {equiv }}$ and $(E A)_{\text {equiv }}$ represent the equivalent bending and tensile stiffness of the cutting tool, respectively; $(\rho I)_{\text {equiv }}$ and $(m)_{\text {equiv }}$ represent the equivalent diametrical mass moment of inertia and the equivalent mass per unit length, respectively; $\rho_{k}$ represents the density of the $k$ th layer; and $r_{\mathrm{k}}$ and $r_{\mathrm{k}+1}$ represent the inner diameter and outer diameter of the $k$ th layer, respectively. The detailed expression of $\bar{Q}_{11}$ can be found in Appendix B. In (9), the primes denote differentiation with respect to $x$.

The following nondimensional quantities are defined:

$$
\begin{gathered}
\bar{x}=\frac{x}{L}, \bar{v}=\frac{v}{L}, \bar{w}=\frac{w}{L}, \bar{t}=\sqrt{\frac{(E I)_{\text {equiv }}}{(m)_{\text {equiv }} L^{4}} t=\gamma t, \bar{\tau}=\gamma \tau, \bar{c}=\frac{c L^{2}}{\sqrt{(m)_{\text {equiv }}(E I)_{\text {equiv }}}},} \\
\bar{\rho} \bar{I}=\frac{(\rho I)_{\text {equiv }}}{\left((m)_{\text {equiv }} L^{2}\right)}, \bar{\Omega}=\sqrt{\frac{(m)_{\text {equiv }} L^{4}}{(E I)_{\text {equiv }}}} \Omega, \lambda=\frac{(E A)_{\text {equiv }} L^{2}}{2(E I)_{\text {equiv }}}, \\
\bar{\alpha}_{0}=\frac{L^{3} \alpha_{0}}{(E I)_{\text {equiv }}}, \bar{\beta}_{0}=\frac{L^{3} \beta_{0}}{(E I)_{\text {equiv }}}, \bar{\gamma}_{0}=\frac{L^{2} \gamma_{0}}{(E I)_{\text {equiv }}}, c_{f \varsigma_{1}}=\frac{L^{2} c_{f}}{(E I)_{\text {equiv }}} \varsigma_{1}, c_{f \eta_{1}}=\frac{L^{2} c_{f}}{(E I)_{\text {equiv }}} \eta_{1}, \\
\alpha_{0}^{\prime}=\frac{L^{3} \alpha_{0}^{\prime}}{(E I)_{\text {equiv }}}, \bar{\beta}_{0}^{\prime}=\frac{L^{3} \beta_{0}^{\prime}}{(E I)_{\text {equiv }}}, \bar{\gamma}_{0}^{\prime}=\frac{L^{2} \gamma_{0}^{\prime}}{(E I)_{\text {equiv }}}, \bar{\varsigma}_{2}=\frac{L^{2} \varsigma_{2}}{(E I)_{\text {equiv }}}, \bar{\eta}_{2}=\frac{L^{2} \eta_{2}}{(E I)_{\text {equiv }}} .
\end{gathered}
$$

Using the above dimensionless variables, (9) is rewritten

as

$$
\begin{aligned}
& \ddot{v}+\bar{c} \dot{v}-2 \bar{\rho} I \bar{\Omega} \dot{w}^{\prime \prime}-\bar{\rho} I \ddot{v}^{\prime \prime}+\frac{\partial^{4} v}{\partial x^{4}}+\lambda\left[\left(v^{\prime^{2}}+w^{\prime^{2}}\right) w^{\prime}\right]^{\prime}=\bar{F}_{y} \delta_{D}(x-1), \\
& \ddot{w}+\bar{c} \dot{w}+2 \bar{\rho} I \bar{\Omega} \dot{v}^{\prime \prime}-\bar{\rho} I \ddot{w}^{\prime \prime}+\frac{\partial^{4} w}{\partial x^{4}}+\lambda\left[\left(v^{\prime^{2}}+w^{\prime^{2}}\right) w^{\prime}\right]^{\prime}=\bar{F}_{z} \delta_{D}(x-1),
\end{aligned}
$$

where

$$
\begin{aligned}
& \bar{F}_{y}=-\frac{N}{2 \pi}\left[\bar{\alpha}_{0}\left(v-v_{\tau}\right)+\bar{\beta}_{0}\left(w-w_{\tau}\right)+\bar{\gamma}_{0}\right]+\frac{1}{2}\left[c_{f \varsigma_{1}} \cos (2 \bar{\Omega} \bar{t}-\pi / 2)-c_{f \eta_{1}} \cos 2 \bar{\Omega} \bar{t}+c_{f \eta_{1}}\right]+\left[\bar{\eta}_{2} \cos (2 \bar{\Omega} \bar{t}-\pi / 2)+\bar{\varsigma}_{2} \cos \bar{\Omega} \bar{t}\right] \\
& \bar{F}_{z}=\frac{N}{2 \pi}\left[\bar{\alpha}_{0}^{\prime}\left(v-v_{\tau}\right)+\bar{\beta}^{\prime}\left(w-w_{\tau}\right)+\gamma_{0}^{\prime}\right]-\frac{1}{2}\left[c_{f \eta_{1}} \cos (2 \bar{\Omega} \bar{t}-\pi / 2)+c_{f \varsigma_{1}} \cos 2 \bar{\Omega} \bar{t}-c_{f \varsigma_{1}}\right]+\left[\bar{\varsigma}_{2} \cos (2 \bar{\Omega} \bar{t}-\pi / 2)-\bar{\eta}_{2} \cos \bar{\Omega} \bar{t}\right] .
\end{aligned}
$$


In (12), the primes denote differentiation with respect to $\bar{x}$, and the superposed dots denote derivatives with respect to the time $\bar{t}$.

The solution of (12) can be written as

$$
v(\bar{x}, \bar{t})=\phi_{1}(\bar{x}) V(\bar{t}), w(\bar{x}, \bar{t})=\phi_{1}(\bar{x}) W(\bar{t}) .
$$

For cantilever beams, $\phi_{1}(\bar{x})$ has to meet the following boundary conditions:

$$
\phi_{1}(0)=\phi_{1}^{\prime}(0)=\phi_{1}^{\prime \prime}(1)=\phi_{1}^{\prime \prime \prime}(1)=0 .
$$

The mode function that satisfies the boundary condition in (12) can be expressed as follows:

$$
\phi_{1}(\bar{x})=\cos \beta_{1} L \bar{x}-\cosh \beta_{1} L \bar{x}-\frac{\cos \beta_{1} L+\cosh \beta_{1} L}{\sin \beta_{1} L+\sinh \beta_{1} L}\left(\sin \beta_{1} L \bar{x}-\sinh \beta_{1} L \bar{x}\right), \bar{x} \in(0,1),
$$

where $\beta_{1} L=1.875$.

Substituting (14) into (9), simplifying the equation by the Galerkin method, and dropping the constant cutting force terms, the following ordinary differential equations can be obtained.

$$
\begin{gathered}
\bar{A}_{1} \ddot{V}+\bar{A}_{2} \dot{V}-\bar{A}_{3} \dot{W}+\bar{A}_{4} V+\bar{A}_{5} V\left(V^{2}+W^{2}\right)=-\frac{N}{2 \pi}\left[\bar{\alpha}_{0}\left(V-V_{\tau}\right)+\bar{\beta}_{0}\left(W-W{ }_{\tau}\right)\right] \phi_{1}^{2}(1)+\bar{F}_{y 2} \phi_{1}(1), \\
\bar{A}_{1} \ddot{W}+\bar{A}_{2} \dot{W}+\bar{A}_{3} \dot{V}+\bar{A}_{4} W+\bar{A}_{5} W\left(V^{2}+W^{2}\right)=\frac{N}{2 \pi}\left[\bar{\alpha}_{0}^{\prime}\left(V-V_{\tau}\right)+\bar{\beta}_{0}^{\prime}(W-W(W)] \phi_{1}^{2}(1)+\bar{F}_{z 2} \phi_{1}(1),\right.
\end{gathered}
$$

where

$$
\begin{aligned}
& \bar{A}_{1}=\int_{0}^{1} \phi_{1}^{2}(\bar{x}) d \bar{x}-\bar{\rho} \bar{I} \int_{0}^{1} \phi_{1}(v) \phi_{1}^{\prime \prime}(\bar{x}) d \bar{x}, \bar{A}_{2}=\bar{c} \int_{0}^{1} \phi_{1}^{2}(\bar{x}) d \bar{x}, \bar{A}_{3}=2 \bar{\rho} \bar{I} \bar{\Omega} \int_{0}^{1} \phi_{1}(\bar{x}) \phi_{1}^{\prime \prime}(\bar{x}) d \bar{x}, \\
& \bar{A}_{4}=\int_{0}^{1} \phi_{1}(\bar{x}) \phi_{1}^{(4)}(\bar{x}) d \bar{x}, \bar{A}_{5}=\lambda \int_{0}^{1}\left(\phi_{1}^{\prime}(\bar{x})^{3}\right)^{\prime} \phi_{1} d \bar{x} .
\end{aligned}
$$

Here, the superscript (4) means the fourth-order partial derivative of $\bar{x}$.

2.4. Perturbation Analysis of Milling Process Using Multiple Scales Method. In order to solve (17) using the multiscale method, the structural damping and nonlinear and exciting force terms are scaled using small parameters $\varepsilon$ as follows:

$$
\begin{aligned}
& \bar{A}_{2} \Rightarrow \varepsilon^{2} \bar{A}_{2}, \frac{N}{2 \pi} \bar{\alpha}_{0} \Rightarrow \varepsilon^{2} \frac{N}{2 \pi} \bar{\alpha}_{0}, \frac{N}{2 \pi} \bar{\beta}_{0} \Rightarrow \varepsilon^{2} \frac{N}{2 \pi} \bar{\beta}_{0}, \frac{N}{2 \pi} \bar{\alpha}_{0}^{\prime} \Rightarrow \varepsilon^{2} \frac{N}{2 \pi} \bar{\alpha}_{0}^{\prime}, \\
& \frac{N}{2 \pi} \bar{\beta}_{0}^{\prime} \Rightarrow \varepsilon^{2} \frac{N}{2 \pi} \bar{\beta}_{0}^{\prime}, \overline{\bar{F}}_{y 2} \Rightarrow \varepsilon^{3} \overline{\bar{F}}_{y 2}, \overline{\bar{F}}_{z 2} \Rightarrow \varepsilon^{3} \overline{\bar{F}}_{z 2} .
\end{aligned}
$$

$V(\bar{t})$ and $W(\bar{t})$ are expanded in the form

$$
\begin{aligned}
V(\bar{t}) & =\varepsilon V_{1}\left(T_{0}, T_{2}\right)+\varepsilon^{3} V_{3}\left(T_{0}, T_{2}\right)+\cdots, \\
W(\bar{t}) & =\varepsilon W_{1}\left(T_{0}, T_{2}\right)+\varepsilon^{3} W_{3}\left(T_{0}, T_{2}\right)+\cdots,
\end{aligned}
$$

where $T_{0}=\bar{t}, T_{2}=\varepsilon^{2} \bar{t}$.

Taking the derivative of (20), one obtains the following equations:

$$
\begin{aligned}
& \dot{V}=\varepsilon \frac{\partial V_{1}}{\partial T_{0}}+\varepsilon^{3}\left(\frac{\partial V_{3}}{\partial T_{0}}+\frac{\partial V_{1}}{\partial T_{2}}\right)+\cdots \\
& \ddot{V}=\varepsilon \frac{\partial^{2} V_{1}}{\partial T_{0}^{2}}+\varepsilon^{3}\left(\frac{\partial^{2} V_{3}}{\partial T_{0}^{2}}+2 \frac{\partial^{2} V_{1}}{\partial T_{0} \partial T_{2}}\right)+\cdots, \\
& \dot{W}=\varepsilon \frac{\partial W_{1}}{\partial T_{0}}+\varepsilon^{3}\left(\frac{\partial W_{3}}{\partial T_{0}}+\frac{\partial W_{1}}{\partial T_{2}}\right)+\cdots \\
& \ddot{W}=\varepsilon \frac{\partial^{2} W_{1}}{\partial T_{0}^{2}}+\varepsilon^{3}\left(\frac{\partial^{2} W_{3}}{\partial T_{0}^{2}}+2 \frac{\partial^{2} W_{1}}{\partial T_{0} \partial T_{2}}\right)+\cdots
\end{aligned}
$$

Substituting (20) and (21) into (17) and considering (19), one can obtain the following equations by comparing the coefficients of $\varepsilon$ and $\varepsilon^{3}$ :

$\mathrm{O}(\varepsilon)$ :

$$
\begin{aligned}
& \bar{A}_{1} \frac{\partial^{2} V_{1}}{\partial T_{0}^{2}}-\bar{A}_{3} \frac{\partial W_{1}}{\partial T_{0}}+\bar{A}_{4} V_{1}=0 \\
& \bar{A}_{1} \frac{\partial^{2} W_{1}}{\partial T_{0}^{2}}+\bar{A}_{3} \frac{\partial V_{1}}{\partial T_{0}}+\bar{A}_{4} W_{1}=0
\end{aligned}
$$


$\mathrm{O}\left(\varepsilon^{3}\right)$ :

$$
\begin{aligned}
& \bar{A}_{1} \frac{\partial^{2} V_{3}}{\partial T_{0}^{2}}-\bar{A}_{3} \frac{\partial W_{3}}{\partial T_{0}}+\bar{A}_{4} V_{3}=-2 \bar{A}_{1} \frac{\partial^{2} V_{1}}{\partial T_{0} \partial T_{2}}-\bar{A}_{2} \frac{\partial V_{1}}{\partial T_{0}}+\bar{A}_{3} \frac{\partial W_{1}}{\partial T_{2}}-\bar{A}_{5} V_{1}\left(V_{1}^{2}+W_{1}^{2}\right)-\frac{N}{2 \pi}\left[\bar{\alpha}_{0}\left(V_{1}-V_{1 \tau}\right)+\bar{\beta}_{0}\left(W_{1}-W_{1 \tau}\right)\right] \\
& \phi_{1}^{2}(1)+\lambda_{V}+\bar{F}_{y 2} \phi_{1}(1), \\
& \bar{A}_{1} \frac{\partial^{2} W_{3}}{\partial T_{0}^{2}}+\bar{A}_{3} \frac{\partial V_{3}}{\partial T_{0}}+\bar{A}_{4} W_{3}=-2 \bar{A}_{1} \frac{\partial^{2} W_{1}}{\partial T_{0} \partial T_{2}}-\bar{A}_{2} \frac{\partial W_{1}}{\partial T_{0}}-\bar{A}_{3} \frac{\partial V_{1}}{\partial T_{2}}-\bar{A}_{5} W_{1}\left(V_{1}^{2}+W_{1}^{2}\right)+\frac{N}{2 \pi}\left[\bar{\alpha}_{0}^{\prime}\left(V_{1}-V_{1 \tau}\right)+\bar{\beta}_{0}^{\prime}\left(W_{1}-W_{1 \tau}\right)\right] \\
& \quad \phi_{1}^{2}(1)+\lambda_{W}+\bar{F}_{z 2} \phi_{1}(1) .
\end{aligned}
$$

Assume the solution of (22) is as follows:

$$
\begin{aligned}
V_{1}\left(T_{0}, T_{2}\right) & =F_{1}\left(T_{2}\right) e^{i \omega_{1} T_{0}}+F_{2}\left(T_{2}\right) e^{i \omega_{2} T_{0}}+\bar{F}_{1}\left(T_{2}\right) e^{-i \omega_{1} T_{0}}+\bar{F}_{2}\left(T_{2}\right) e^{-i \omega_{2} T_{0}}, \\
W_{1}\left(T_{0}, T_{2}\right) & =-i F_{1}\left(T_{2}\right) e^{i \omega_{1} T_{0}}+i F_{2}\left(T_{2}\right) e^{i \omega_{2} T_{0}}+i \bar{F}_{1}\left(T_{2}\right) e^{-i \omega_{1} T_{0}}-i \bar{F}_{2}\left(T_{2}\right) e^{-i \omega_{2} T_{0}}, \\
V_{1 \tau}\left(T_{0}, T_{2}\right) & =F_{1}\left(T_{2}\right) e^{i \omega_{1}\left(T_{0}-\bar{\tau}\right)}+F_{2}\left(T_{2}\right) e^{i \omega_{2}\left(T_{0}-\bar{\tau}\right)}+\bar{F}_{1}\left(T_{2}\right) e^{-i \omega_{1}\left(T_{0}-\bar{\tau}\right)}+\bar{F}_{2}\left(T_{2}\right) e^{-i \omega_{2}\left(T_{0}-\bar{\tau}\right)}, \\
W_{1 \tau}\left(T_{0}, T_{2}\right) & =-i F_{1}\left(T_{2}\right) e^{i \omega_{1}\left(T_{0}-\bar{\tau}\right)}+i F_{2}\left(T_{2}\right) e^{i \omega_{2}\left(T_{0}-\bar{\tau}\right)}+i \bar{F}_{1}\left(T_{2}\right) e^{-i \omega_{1}\left(T_{0}-\bar{\tau}\right)}-i \bar{F}_{2}\left(T_{2}\right) e^{-i \omega_{2}\left(T_{0}-\bar{\tau}\right),}
\end{aligned}
$$

where $i=\sqrt{-1}$ represents the imaginary unit, $F_{1}\left(T_{2}\right)$ and $F_{2}\left(T_{2}\right)$ are the complex functions to be determined, and $\bar{F}_{1}\left(T_{2}\right)$ and $\bar{F}_{2}\left(T_{2}\right)$ represent the complex conjugate. $\omega_{1}$ and $\omega_{2}$ refer to the forward and backward whirling frequencies, respectively, which are expressed as follows:

$$
\omega_{1}=\frac{\bar{A}_{3}+\sqrt{\bar{A}_{3}^{2}+4 \bar{A}_{1} \bar{A}_{4}}}{2 \bar{A}_{1}}, \omega_{2}=\frac{-\bar{A}_{3}+\sqrt{\bar{A}_{3}^{2}+4 \bar{A}_{1} \bar{A}_{4}}}{2 \bar{A}_{1}} .
$$

Substituting (24) into (23) and dropping the constant terms $\lambda_{V}$ and $\lambda_{W}$, one obtains the following equations:

$$
\begin{aligned}
& \bar{A}_{1} \frac{\partial^{2} V_{3}}{\partial T_{0}^{2}}-\bar{A}_{3} \frac{\partial W_{3}}{\partial T_{0}}+\bar{A}_{4} V_{3}=P_{3} e^{i \omega_{1} T_{0}}+Q_{3} e^{i \omega_{2} T_{0}}+\overline{\bar{F}}_{y 2}, \\
& \bar{A}_{1} \frac{\partial^{2} W_{3}}{\partial T_{0}^{2}}+\bar{A}_{3} \frac{\partial V_{3}}{\partial T_{0}}+\bar{A}_{4} W_{3}=P_{4} e^{i \omega_{1} T_{0}}+Q_{4} e^{i \omega_{2} T_{0}}+\overline{\bar{F}}_{z 2},
\end{aligned}
$$

where

$$
\begin{aligned}
P_{3} & =\Gamma_{1}-i \Lambda_{1} F_{1}^{\prime}-i \bar{A}_{2} \omega_{1} F_{1}-\phi_{1}^{2}(1) \frac{N}{2 \pi}\left(\overline{\bar{\alpha}}_{0}-i \overline{\bar{\beta}}_{0}\right)\left(1-e^{-i \omega_{1} \bar{\tau}}\right) F_{1} \\
Q_{3} & =\Gamma_{2}-i \Lambda_{2} F_{2}^{\prime}-i \bar{A}_{2} \omega_{2} F_{2}-\phi_{1}^{2}(1) \frac{N}{2 \pi}\left(\overline{\bar{\alpha}}_{0}+i \overline{\bar{\beta}}_{0}\right)\left(1-e^{-i \omega_{2} \bar{\tau}}\right) F_{2} \\
P_{4} & =-i \Gamma_{1}-\Lambda_{1} F_{1}^{\prime}-\bar{A}_{2} \omega_{1} F_{1}+\phi_{1}^{2}(1) \frac{N}{2 \pi}\left(\overline{\bar{\alpha}}_{0}^{\prime}-i \overline{\bar{\beta}}_{0}^{\prime}\right)\left(1-e^{-i \omega_{1} \bar{\tau}}\right) F_{1} \\
Q_{4} & =i \Gamma_{2}+\Lambda_{2} F_{2}^{\prime}+\bar{A}_{2} \omega_{2} F_{2}+\phi_{1}^{2}(1) \frac{N}{2 \pi}\left(\overline{\bar{\alpha}}_{0}^{\prime}+i \overline{\bar{\beta}}_{0}^{\prime}\right)\left(1-e^{-i \omega_{2} \bar{\tau}}\right) F_{2}, \\
\Lambda_{1} & =2 \bar{A}_{1} \omega_{1}+\bar{A}_{3}, \Gamma_{1}=-4 \bar{A}_{5}\left(F_{1}^{2} \bar{F}_{1}+2 F_{1} F_{2} \bar{F}_{2}\right) \\
\Lambda_{2} & =2 \bar{A}_{1} \omega_{2}-\bar{A}_{3}, \Gamma_{2}=-4 \bar{A}_{5}\left(F_{2}^{2} \bar{F}_{2}+2 F_{1} F_{2} \bar{F}_{1}\right) \\
\overline{\bar{F}}_{y_{2}} & =\bar{F}_{y_{2}} \phi_{1}(1) \\
\bar{F}_{z_{2}} & =\bar{F}_{z_{2}} \phi_{1}(1)
\end{aligned}
$$


In this paper, four cases of primary and superharmonic resonances, i.e., case I: $\bar{\Omega}=\omega_{1}$, case II: $\bar{\Omega}=\omega_{2}$, case III: $2 \bar{\Omega}=\omega_{1}$, and case IV: $2 \bar{\Omega}=\omega_{2}$, are studied.

2.4.1. Case I: Primary Resonance $\left(\bar{\Omega}=\omega_{1}\right)$. Assume

$$
\bar{\Omega}=\omega_{1}+\varepsilon^{2} \sigma
$$

where $\sigma$ represents the detuning parameter.

Substituting (24) into (26), one can obtain the following equations:

$$
\begin{aligned}
& \bar{A}_{1} \frac{\partial^{2} V_{3}}{\partial T_{0}^{2}}-\bar{A}_{3} \frac{\partial W_{3}}{\partial T_{0}}+\bar{A}_{4} V_{3}=\bar{P}_{3} e^{i \omega_{1} T_{0}}+\bar{Q}_{3} e^{i \omega_{2} T_{0}} \\
& \bar{A}_{1} \frac{\partial^{2} W_{3}}{\partial T_{0}^{2}}+\bar{A}_{3} \frac{\partial V_{3}}{\partial T_{0}}+\bar{A}_{4} W_{3}=\bar{P}_{4} e^{i \omega_{1} T_{0}}+\bar{Q}_{4} e^{i \omega_{2} T_{0}}
\end{aligned}
$$

where

$$
\begin{aligned}
& \bar{P}_{3}=P_{3}+\phi_{1}(1) \bar{q}_{1} e^{i \sigma T_{2}}, \\
& \bar{P}_{4}=P_{4}-i \phi_{1}(1) \bar{q}_{1} e^{i \sigma T_{2}}, \\
& \bar{Q}_{3}=Q_{3}, \\
& \bar{Q}_{4}=Q_{4},
\end{aligned}
$$

where $\bar{q}_{1}=\left(\bar{\varsigma}_{2}-i \bar{\eta}_{2}\right) / 2$.

The particular solutions of (29) are

$$
\begin{gathered}
V_{3}\left(T_{0}, T_{2}\right)=F_{11}\left(T_{2}\right) e^{i \omega_{1} T_{0}}+F_{12}\left(T_{2}\right) e^{i \omega_{2} T_{0}}, \\
W_{3}\left(T_{0}, T_{2}\right)=F_{21}\left(T_{2}\right) e^{i \omega_{1} T_{0}}+F_{22}\left(T_{2}\right) e^{i \omega_{2} T_{0}} .
\end{gathered}
$$

Substituting (31) into (29) and equating the coefficient of $e^{i \omega_{1} T_{0}}$ and $e^{i \omega_{2} T_{0}}$ in both sides of (29), one has

$$
\begin{aligned}
& \left(\bar{A}_{4}-\bar{A}_{1} \omega_{1}^{2}\right) F_{11}-i \bar{A}_{3} \omega_{1} F_{21}=\bar{P}_{3}, \\
& i \bar{A}_{3} \omega_{1} F_{11}+\left(\bar{A}_{4}-\bar{A}_{1} \omega_{1}^{2}\right) F_{21}=\bar{P}_{4} . \\
& \left(\bar{A}_{4}-\bar{A}_{1} \omega_{2}^{2}\right) F_{12}-i \bar{A}_{3} \omega_{2} F_{22}=\bar{Q}_{3}, \\
& i \bar{A}_{3} \omega_{2} F_{12}+\left(\bar{A}_{4}-\bar{A}_{1} \omega_{2}^{2}\right) F_{22}=\bar{Q}_{4} .
\end{aligned}
$$

Equations (32) and (33) constitute systems of two inhomogeneous algebraic equations for $F_{11}, F_{21}$ and $F_{12}, F_{22}$, respectively. Their homogeneous parts have a nontrivial solution. Then their solvability conditions can be written as [36]

$$
\begin{aligned}
& \left|\begin{array}{cc}
\bar{P}_{3} & -i \bar{A}_{3} \omega_{1} \\
\bar{P}_{4} & \bar{A}_{4}-\bar{A}_{1} \omega_{1}^{2}
\end{array}\right|=0, \\
& \left|\begin{array}{cc}
\bar{A}_{4}-\bar{A}_{1} \omega_{1}^{2} & \bar{P}_{3} \\
i \bar{A}_{3} \omega_{1} & \bar{P}_{4}
\end{array}\right|=0 . \\
& \left|\begin{array}{cc}
\bar{Q}_{3} & -i \bar{A}_{3} \omega_{2} \\
\bar{Q}_{4} & \bar{A}_{4}-\bar{A}_{1} \omega_{2}^{2}
\end{array}\right|=0, \\
& \left|\begin{array}{cc}
\bar{A}_{4}-\bar{A}_{1} \omega_{2}^{2} & \bar{Q}_{3} \\
i \bar{A}_{3} \omega_{2} & \bar{Q}_{4}
\end{array}\right|=0 .
\end{aligned}
$$

After simplification, the solvability conditions are reduced to two independent governing equations in the following form:

$$
\begin{gathered}
4 \bar{A}_{5}\left(F_{1}^{2} \bar{F}_{1}+2 F_{1} F_{2} \bar{F}_{2}\right)-i \Lambda_{1} F_{1}^{\prime}-i \bar{A}_{2} \omega_{1} F_{1}+\phi_{1}^{2}(1) \frac{N}{4 \pi} \\
{\left[\bar{\beta}_{0}^{\prime}-\bar{\alpha}_{0}+i\left(\bar{\alpha}_{0}^{\prime}+\bar{\beta}_{0}\right)\right]\left(1-e^{-i \omega_{1} \bar{\tau}}\right) F_{1}+\phi_{1}(1) \bar{q}_{1} e^{i \sigma T_{2}}=0,} \\
4 \bar{A}_{5}\left(F_{2}^{2} \bar{F}_{2}+2 F_{1} F_{2} \bar{F}_{1}\right)-i \Lambda_{2} F_{2}^{\prime}-i \bar{A}_{2} \omega_{2} F_{2}-\frac{1}{2} \phi_{1}^{2}(1) \frac{N}{2 \pi} \\
{\left[\bar{\alpha}_{0}+\bar{\beta}_{0}^{\prime}+i\left(\bar{\alpha}_{0}^{\prime}-\bar{\beta}_{0}\right)\right]\left(1-e^{-i \omega_{1} \bar{\tau}}\right) F_{2}=0 .}
\end{gathered}
$$

Assume that the solutions of (36) and (37) are

$$
\begin{aligned}
& F_{1}=\frac{1}{2} a_{1}\left(T_{2}\right) e^{i \theta_{1}\left(T_{2}\right),} \\
& F_{2}=\frac{1}{2} a_{2}\left(T_{2}\right) e^{i \theta_{2}\left(T_{2}\right)},
\end{aligned}
$$

where $a_{j}\left(T_{2}\right)$ and $\theta_{j}\left(T_{2}\right)(j=1,2)$ refer to the amplitudes and phase angles of the response, respectively.

Substituting (38) into (36) and (37), separating the real and the imaginary parts, one can obtain the following equations:

$$
\begin{gathered}
\Lambda_{1} a_{1}^{\prime}=-\bar{A}_{2} \omega_{1} a_{1}+\phi_{1}^{2}(1) \frac{N}{4 \pi}\left(\left(\bar{\alpha}_{0}^{\prime}+\bar{\beta}_{0}\right)\left(1-\cos \omega_{1} \bar{\tau}\right)+\left(\bar{\beta}_{0}^{\prime}-\bar{\alpha}_{0}\right) \sin \omega_{1} \bar{\tau}\right) a_{1}+\phi_{1}(1)\left(\bar{\varsigma}_{2} \sin \psi_{1}-\bar{\eta}_{2} \cos \psi_{1}\right) \\
\Lambda_{1} a_{1} \psi_{1}^{\prime}=\Lambda_{1} \sigma a_{1}-\bar{A}_{5}\left(a_{1}^{3}+2 a_{1} a_{2}^{2}\right)+\phi_{1}^{2}(1) \frac{N}{4 \pi}\left(\left(\bar{\beta}_{0}^{\prime}-\bar{\alpha}_{0}\right)\left(1-\cos \omega_{1} \bar{\tau}\right)-\left(\bar{\alpha}_{0}^{\prime}+\bar{\beta}_{0}\right) \sin \omega_{1} \bar{\tau}\right) a_{1}+\phi_{1}(1)\left(\bar{\eta}_{2} \sin \psi_{1}+\bar{\varsigma}_{2} \cos \psi_{1}\right) \\
\Lambda_{2} a_{2}^{\prime}=-\bar{A}_{2} \omega_{2} a_{2}-\phi_{1}^{2}(1) \frac{N}{4 \pi}\left(\left(\bar{\alpha}_{0}^{\prime}+\bar{\beta}_{0}\right)\left(1-\cos \omega_{2} \bar{\tau}\right)+\left(\bar{\alpha}_{0}-\bar{\beta}_{0}^{\prime}\right) \sin \omega_{2} \bar{\tau}\right) a_{2} \\
\Lambda_{2} \psi_{2}^{\prime} a_{2}=-\bar{A}_{5}\left(a_{2}^{3}+2 a_{1}^{2} a_{2}\right)-\phi_{1}^{2}(1) \frac{N}{4 \pi}\left(\left(\bar{\alpha}_{0}-\bar{\beta}_{0}^{\prime}\right)\left(1-\cos \omega_{2} \bar{\tau}\right)-\left(\bar{\alpha}_{0}^{\prime}+\bar{\beta}_{0}\right) \sin \omega_{2} \bar{\tau}\right) a_{2}
\end{gathered}
$$


where $\sigma T_{2}-\theta_{j}=\psi_{j}(j=1,2)$.

To determine the steady-state forced response, the time derivatives in (39) and (40) are set to zero. It can be immediately concluded from (40) that only solution for $a_{2}$ is zero. This shows that under the primary resonance $\Omega \approx \omega_{1}$, only the first mode, i.e., the forward mode, can be excited, while the second mode, i.e., the backward mode, does not participate in the primary resonance and remains stationary.

Substituting $a_{2}=0$ into (39) and solving for $\sigma$, one obtains

$$
\begin{aligned}
\sigma= & -\phi_{1}^{2}(1) \frac{N}{4 \pi \Lambda_{1}}\left[\left(\bar{\beta}_{0}^{\prime}-\bar{\alpha}_{0}\right)\left(1-\cos \omega_{1} \bar{\tau}\right)-\left(\bar{\alpha}_{0}^{\prime}+\bar{\beta}_{0}\right) \sin \omega_{1} \bar{\tau}\right]+\frac{\bar{A}_{5}}{\Lambda_{1}} a_{1}^{2} \\
& \pm \frac{1}{\Lambda_{1}} \sqrt{\frac{\phi_{1}^{2}(1)\left(\bar{\zeta}_{2}^{2}+\bar{\eta}_{2}^{2}\right)}{a_{1}^{2}}-\left[\bar{A}_{2} \omega_{1}-\phi_{1}^{2}(1) \frac{N}{4 \pi}\left[\left(\bar{\alpha}_{0}^{\prime}+\bar{\beta}_{0}\right)\left(1-\cos \omega_{1} \bar{\tau}\right)+\left(\bar{\beta}_{0}^{\prime}-\bar{\alpha}_{0}\right) \sin \omega_{1} \bar{\tau}\right]\right]^{2}} .
\end{aligned}
$$

To study the stability of the steady-state response of case I, the nature of steady-state response can be investigated numerically by linearizing (39) (with $a_{2}=0$ ) around $\left(a_{0}, \psi_{0}\right)$.

$$
\bar{\Omega}=\omega_{2}+\varepsilon^{2} \sigma .
$$

Using their solvability conditions (which are not shown) and (38) leads to

2.4.2. Case II: Primary Resonance $\left(\bar{\Omega}=\omega_{2}\right)$. According to (26), under such conditions, one introduces detuning parameters as

$$
\begin{aligned}
\Lambda_{1} a_{1}^{\prime}= & -\bar{A}_{2} \omega_{1} a_{1}+\phi_{1}^{2}(1) \frac{N}{4 \pi}\left(\left(\bar{\alpha}_{0}^{\prime}+\bar{\beta}_{0}\right)\left(1-\cos \omega_{1} \bar{\tau}\right)+\left(\bar{\beta}_{0}^{\prime}-\bar{\alpha}_{0}\right) \sin \omega_{1} \bar{\tau}\right) a_{1}, \\
\Lambda_{1} a_{1} \psi_{1}^{\prime}= & \Lambda_{1} \sigma a_{1}-\bar{A}_{5}\left(a_{1}^{3}+2 a_{1} a_{2}^{2}\right)+\phi_{1}^{2}(1) \frac{N}{4 \pi}\left(\left(\bar{\beta}_{0}^{\prime}-\bar{\alpha}_{0}\right)\left(1-\cos \omega_{1} \bar{\tau}\right)-\left(\bar{\alpha}_{0}^{\prime}+\bar{\beta}_{0}\right) \sin \omega_{1} \bar{\tau}\right) a_{1}, \\
\Lambda_{2} a_{2}^{\prime}= & -\bar{A}_{2} \omega_{2} a_{2}-\phi_{1}^{2}(1) \frac{N}{4 \pi}\left(\left(\bar{\alpha}_{0}^{\prime}+\bar{\beta}_{0}\right)\left(1-\cos \omega_{2} \bar{\tau}\right)+\left(\bar{\alpha}_{0}-\bar{\beta}_{0}^{\prime}\right) \sin \omega_{2} \bar{\tau}\right) a_{2}-\phi_{1}(1)\left(\bar{\varsigma}_{2} \sin \psi_{2}-\bar{\eta}_{2} \cos \psi_{2}\right), \\
\Lambda_{2} \psi_{2}^{\prime} a_{2} & =-\bar{A}_{5}\left(a_{2}^{3}+2 a_{1}^{2} a_{2}\right)-\phi_{1}^{2}(1) \frac{N}{4 \pi}\left(\left(\bar{\alpha}_{0}-\bar{\beta}_{0}^{\prime}\right)\left(1-\cos \omega_{2} \bar{\tau}\right)-\left(\bar{\alpha}_{0}^{\prime}+\bar{\beta}_{0}\right) \sin \omega_{2} \bar{\tau}\right) a_{2}-\phi_{1}(1)\left(\bar{\varsigma}_{2} \cos \psi_{2}+\bar{\eta}_{2} \sin \psi_{2}\right) .
\end{aligned}
$$

By using steady-state condition (i.e., set $a_{1}^{\prime}=0, \psi_{1}^{\prime}=0, a_{2}^{\prime}$ $=0$, and $\psi_{2}^{\prime}=0$ ), the following steady-state response of case II can be obtained:

$$
\begin{aligned}
\sigma= & \phi_{1}^{2}(1) \frac{N}{4 \pi \Lambda_{2}}\left[\left(\bar{\alpha}_{0}-\bar{\beta}_{0}^{\prime}\right)\left(1-\cos \omega_{2} \bar{\tau}\right)-\left(\bar{\alpha}_{0}^{\prime}+\bar{\beta}_{0}\right) \sin \omega_{2} \bar{\tau}\right]+\frac{\bar{A}_{5}}{\Lambda_{2}} a_{2}^{2} \\
& \pm \frac{1}{\Lambda_{2}} \sqrt{\frac{\phi_{1}^{2}(1)\left(\bar{\varsigma}_{2}^{2}+\bar{\eta}_{2}^{2}\right)}{a_{2}^{2}}-\left[\frac{2}{A} \omega_{2}+\phi_{1}^{2}(1) \frac{N}{4 \pi}\left[\left(\bar{\alpha}_{0}^{\prime}+\bar{\beta}_{0}\right)\left(1-\cos \omega_{2} \bar{\tau}\right)+\left(\bar{\alpha}_{0}-\bar{\beta}_{0}^{\prime}\right) \sin \omega_{2} \bar{\tau}\right]\right]^{2}} .
\end{aligned}
$$

From (44), it can be seen that $a_{1}=0$ and $a_{2} \neq 0$, indicating that only the forced vibration response of backward mode is excited, while forward mode remains stationary.
2.4.3. Case III: Superharmonic Resonance $\left(2 \bar{\Omega}=\omega_{1}\right)$. For this case, the formulation of steady-state response is the same as case I, but in (41), $\bar{\varsigma}_{2}$ and $\bar{\eta}_{2}$ are replaced with $c_{f \varsigma_{1}} / 2$ and $c_{f \eta_{1}} / 2$, respectively. 
2.4.4. Case IV: Superharmonic Resonance $\left(2 \bar{\Omega}=\omega_{2}\right)$. For this case, the formulation of steady-state response is the same as case II, but in (43), $\bar{\varsigma}_{2}$ and $\bar{\eta}_{2}$ are replaced with $c_{f_{\varsigma_{1}}} / 2$ and $c_{f \eta_{1}} / 2$, respectively.

\section{Numerical Results and Discussions}

In this study, the composite material of carbon fiber/epoxy resin is selected as the material of the cutting tool. The mechanical properties of the material are shown in Table 1. Coefficients of cutting forces for simulation are given in Table 2. The cutting tool has a hollow structure, the outer diameter of the cross section is $D=8 \mathrm{~mm}$, the inner diameter is $D=4 \mathrm{~mm}$, the thickness of the section is $h=2 \mathrm{~mm}$, and the length $L$ is determined by the given ratio of length to diameter. The composite cutting tool has 16 layers with identical thickness, and the stack sequence is $[ \pm \theta]_{8}$. In all cases, $\bar{\Omega}=200$, except where other values are mentioned.

Figure 2 shows the natural frequency versus rotating speed, which is generally known as a Campbell diagram. In vibration of gyroscopic systems, there are two natural frequencies associated with forward and backward whirling motions. In forward natural frequency, the natural frequency is measured when the rotating cutting tool whirls in direction of the rotation. However, in backward natural frequency, the natural frequency is measured when the cutting tool whirls in the opposite direction of the rotation. The forward natural frequency (black solid line) increases with the increase of the rotating speed, while the backward natural frequency (blue dashed line) decreases with the increase of the rotating speed. The intersections of the curves related to the natural frequencies with the straight line $\omega_{n}=$ $\bar{\Omega}$ determine the critical rotating speeds of the rotating cutting tool.

3.1. Stability Lobe Diagram. By removing the nonlinear term and the harmonic cutting force in the right-side term of (17), the stability of the cutting system can be investigated. Figure 3 shows a stability lobe diagram where the $\Omega$ versus $a_{\text {lim }}$ curve separates the space into two regions. Any $\left(\Omega, a_{\text {lim }}\right)$ pair that appears above the collective boundary indicates unstable milling process where regenerative chatter or selfexcited vibration occurs, while any pair below the boundary is a stable milling process.

\subsection{Primary Resonance Response}

3.2.1. Case I. The numerical solutions for the forced vibration responses of the cutting system with composite cutting tool are presented in Figure 4. As shown in Figure 4, by increasing detuning parameters at point $\mathrm{A}$, the amplitude $a_{1}$ gradually increases until reaching point $\mathrm{B} . a_{1}$ jumps downward from point B to point C. Afterward, $a_{1}$ gradually drops to point $D$ while continuing increasing the detuning parameter. On the other hand, as the detuning parameter decreases from point $D$ to point $E, a_{1}$ increases and jumps upward from point $E$ to point $\mathrm{F}$. As the detuning parameter further decreases, $a_{1}$ drops until arriving at point A. By
TABLE 1: Mechanical properties of carbon fiber/epoxy composite [37].

\begin{tabular}{lccccc}
\hline$\rho\left(\mathrm{kg} / \mathrm{m}^{3}\right)$ & $E_{11}(\mathrm{GPa})$ & $E_{22}(\mathrm{GPa})$ & $G_{12}(\mathrm{GPa})$ & $G_{23}(\mathrm{GPa})$ & $\nu_{12}$ \\
\hline 1672 & 25.8 & 8.7 & 3.5 & 3.5 & 0.34 \\
\hline
\end{tabular}

TABLE 2: Coefficients of cutting forces [21].

\begin{tabular}{lcccccc}
\hline $\begin{array}{l}C_{f}(\mathrm{~mm} / \mathrm{rev}- \\
\text { tooth })\end{array}$ & $\begin{array}{c}\overline{\bar{\zeta}}_{1}(\mathrm{~N} / \\
\mathrm{mm})\end{array}$ & $\begin{array}{c}\overline{\bar{\eta}}_{1} \\
(\mathrm{~N})\end{array}$ & $\begin{array}{c}\overline{\bar{\varsigma}}_{2}(\mathrm{~N} / \\
\mathrm{mm})\end{array}$ & $\begin{array}{c}\overline{\bar{\eta}}_{2} \\
(\mathrm{~N})\end{array}$ & $\begin{array}{c}a_{c} \\
(\mathrm{~mm})\end{array}$ & $N$ \\
\hline 0.2 & 620 & 208 & 43 & 52 & 3 & 4 \\
\hline
\end{tabular}

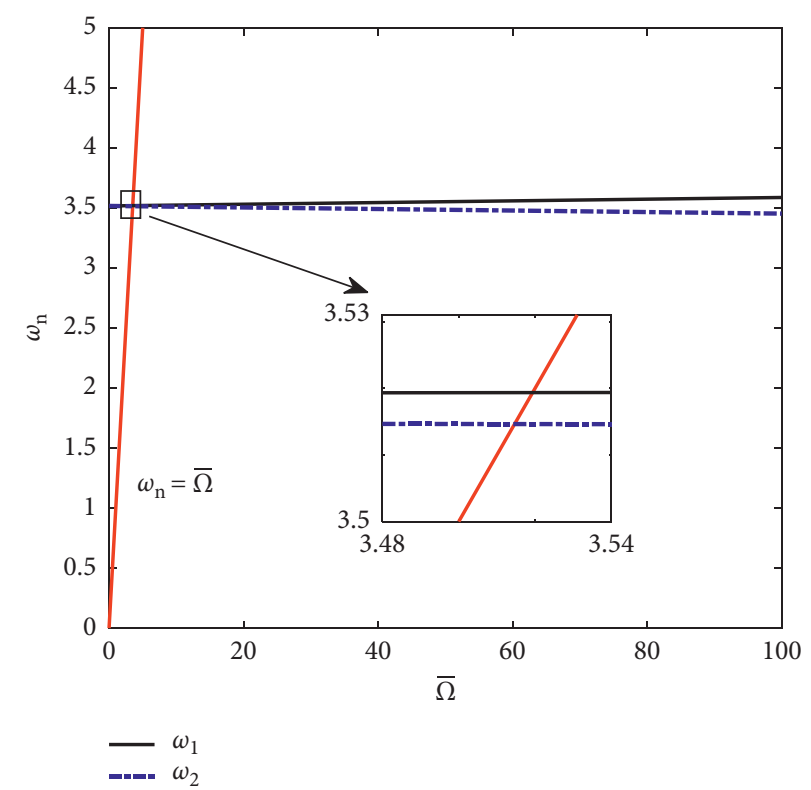

Figure 2: Natural frequency versus rotational speed $(\mathrm{L} / \mathrm{d}=10$, $\theta=0^{\circ}$ ).

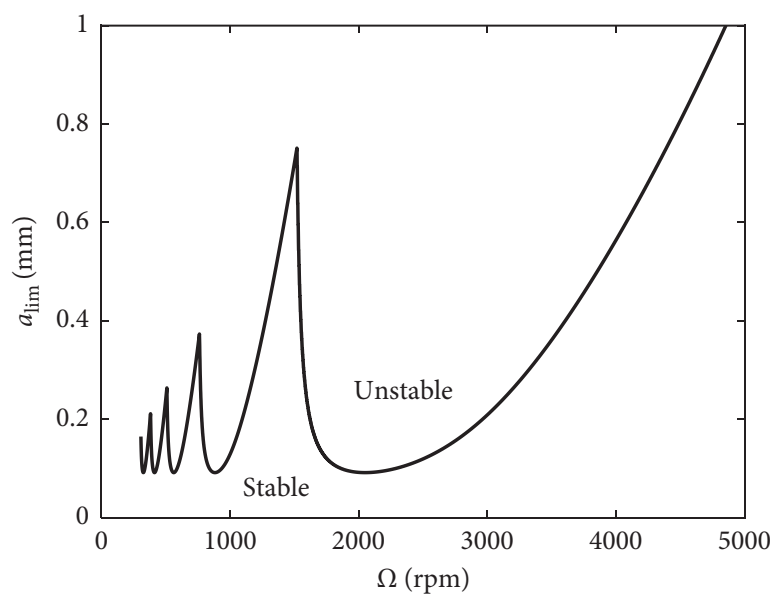

FIgURE 3: Stability lobe diagram of the cutting system with composite cutting tool $\left(\mathrm{L} / \mathrm{d}=10, \bar{c}=0.3, \theta=0^{\circ}\right)$.

considering the nonlinearity of higher-order bending deformation, the forced response curve of the cutting system deviates toward the right, suggesting hard spring vibration 


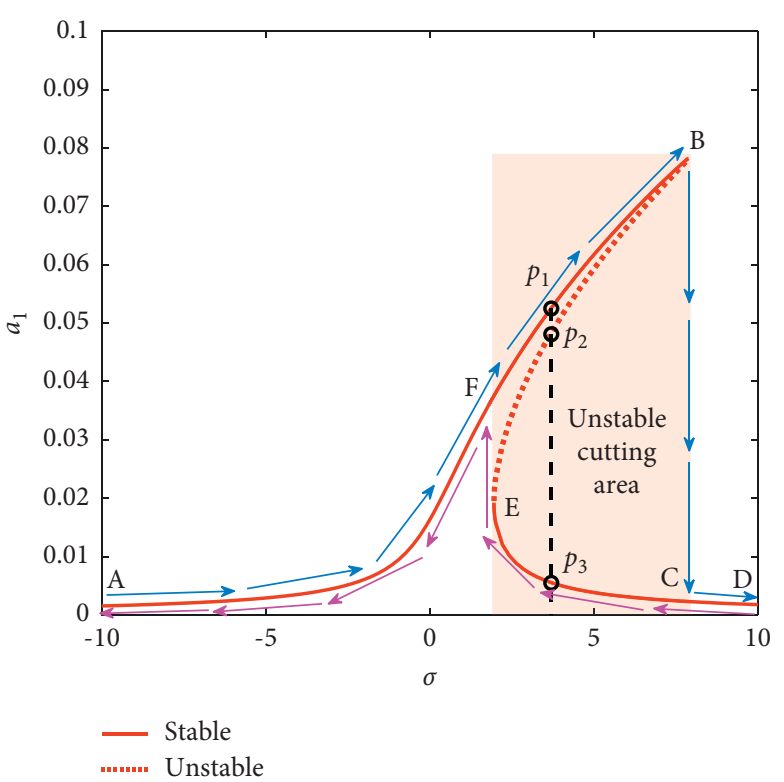

Figure 4: Frequency response curve in case I.

behavior of Duffing type oscillator. As the detuning parameter varies between $\sigma_{F}$ and $\sigma_{B}$, the cutting system has three steady-state vibration amplitudes, $p_{1}, p_{2}$, and $p_{3}$, among which $p_{1}$ and $p_{3}$ are stable and $p_{2}$ is unstable. This means that the cutting system works in an unstable cutting state. Therefore, cutting conditions and consequently initial conditions should be adjusted such that the stable branch with less vibration amplitudes is realized in practice.

Figures 5-9 show the effects of ratio of length to diameter, structural damping, cutting force, ply angle, and rotating speed on frequency response curve, respectively, for the case $\bar{\Omega}=\omega_{1}$. As shown in Figure 5, when ratio of length to diameter increases, vibration amplitudes decrease and frequency response curve bends more strongly toward left. This is physically expected, because the nonlinear stiffness coefficient $\lambda=(E A)_{\text {equiv }} L^{2} / 2(E I)_{\text {equiv }}$ is proportional to ratio of length to diameter according to (12). As is observed and physically expected from Figure 6, the increase in the structural damping leads to the decrease in vibration amplitudes. In order to study the effect of cutting force coefficients, we define $\varsigma_{2}=K_{f} \overline{\bar{\zeta}}_{2}, \eta_{2}=K_{f} \overline{\bar{\eta}}_{2}$, with the parameters $\overline{\bar{\zeta}}_{2}$ and $\overline{\bar{\eta}}_{2}$ given in Table 2. As is shown in Figure 7, by increasing cutting forces, vibration amplitudes increase. Figure 8 shows that the primary resonance amplitude increases with the increase of the ply angle because the elastic modulus $E_{11}$ along the longitudinal direction of the fiber is significantly larger than the transverse elastic modulus $E_{22}$ (as shown in Table 1). Therefore, as the ply angle is greater, the equivalent bending stiffness (EI) equiv of the composite cutting tool is smaller; thus, the nondimensional cutting force coefficients are greater (as shown in (11)). As a result, the amplitude of the primary resonance response is larger.

Figure 9 shows that vibration amplitudes decrease with the decrease of rotating speed. This is physically expected, because the equivalent damping of the cutting system (which will be introduced in the subsequent section) increases with

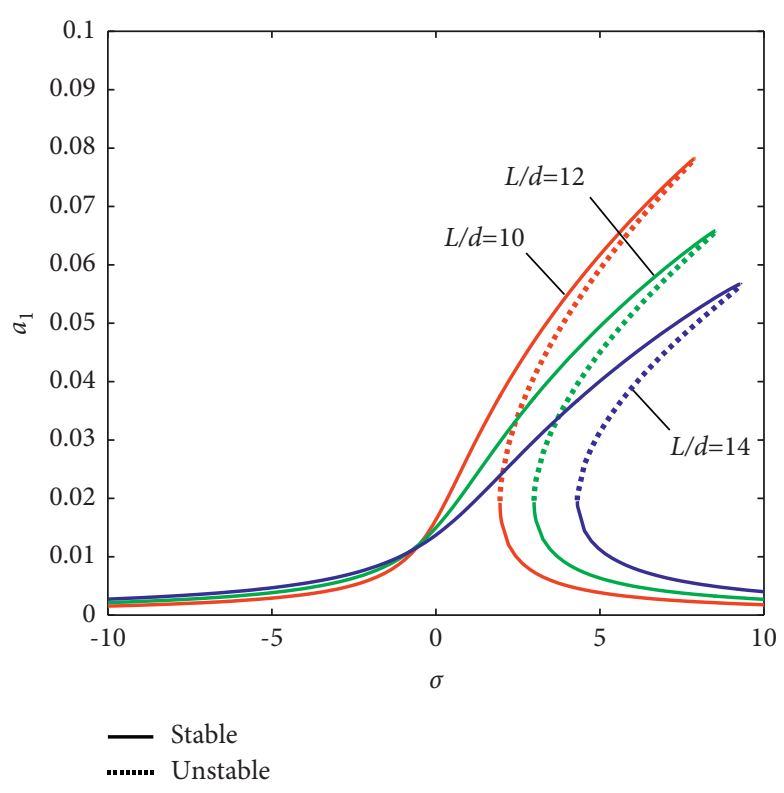

Figure 5: The effect of ratio of length to diameter on frequency response curve $\left(\bar{c}=0.01, \theta=0^{\circ}\right.$, case I).

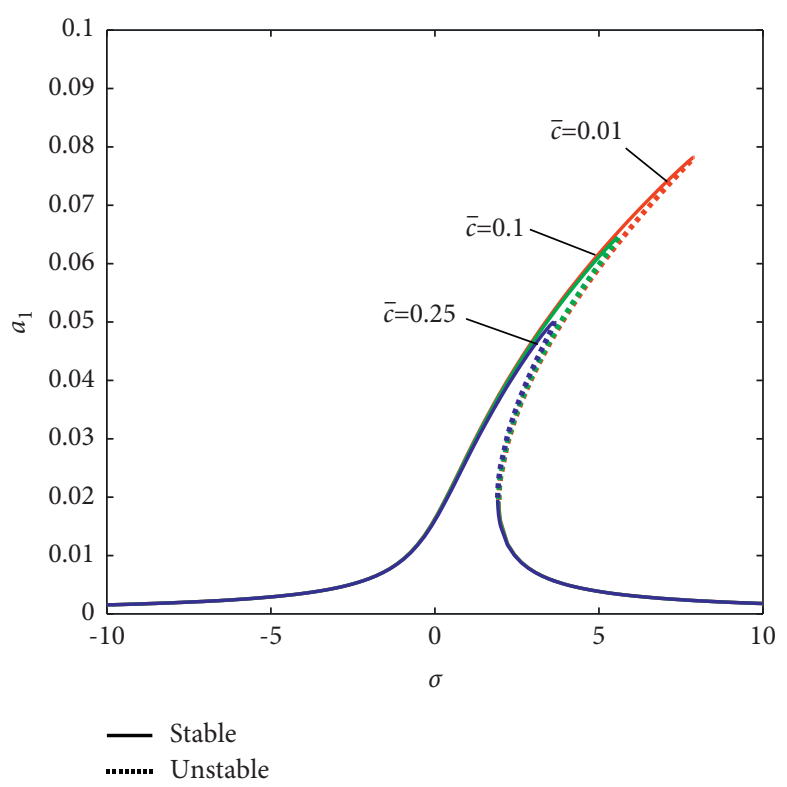

Figure 6: The effect of structural damping on frequency response curve $\left(\mathrm{L} / \mathrm{d}=10, \theta=0^{\circ}\right.$, case $\left.\mathrm{I}\right)$.

the decrease of rotating speed due to the damping effect from regenerative chatter mechanism.

Figures 10-14 show the amplitude versus damping coefficient with different ratios of length to diameter, detuning parameter values, cutting force coefficients, ply angles, and rotating speeds, respectively, for the case $\bar{\Omega}=\omega_{1}$. From these figures, it can be seen that for some values of $L / d, \sigma, K_{f}$, or $\theta$, there are multivalued curves. For example, when $L / d=10$ and $\bar{c}<0.45$, as is shown in Figure 10, the system has two stable and one unstable branches, but for $L / d=10$ and $\bar{c}>0.45$, there exists only 


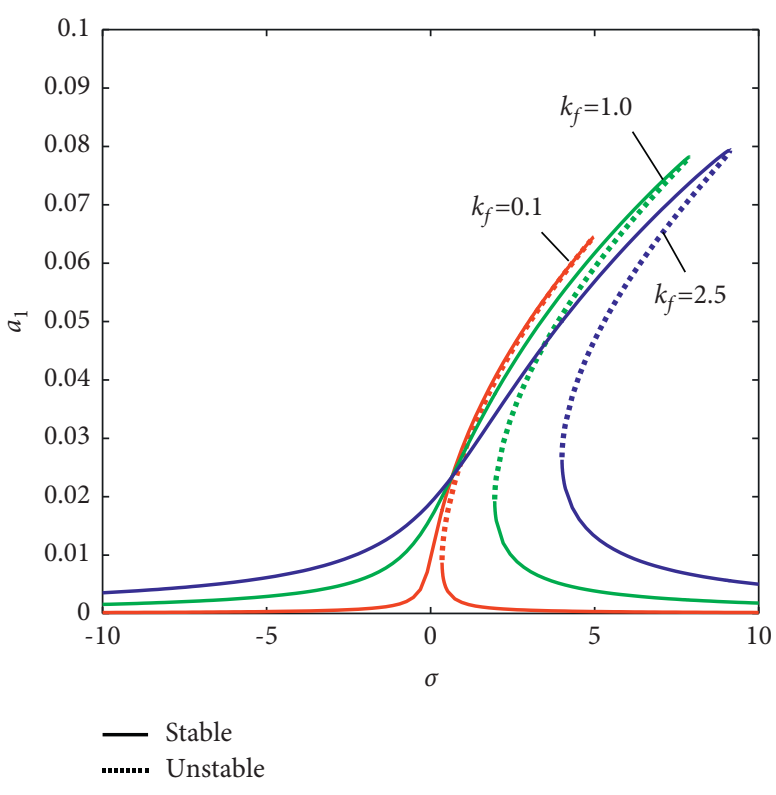

Figure 7: The effect of cutting force coefficient on frequency response curve $\left(\mathrm{L} / \mathrm{d}=10, \bar{c}=0.01, \theta=0^{\circ}\right.$, case $\left.\mathrm{I}\right)$.

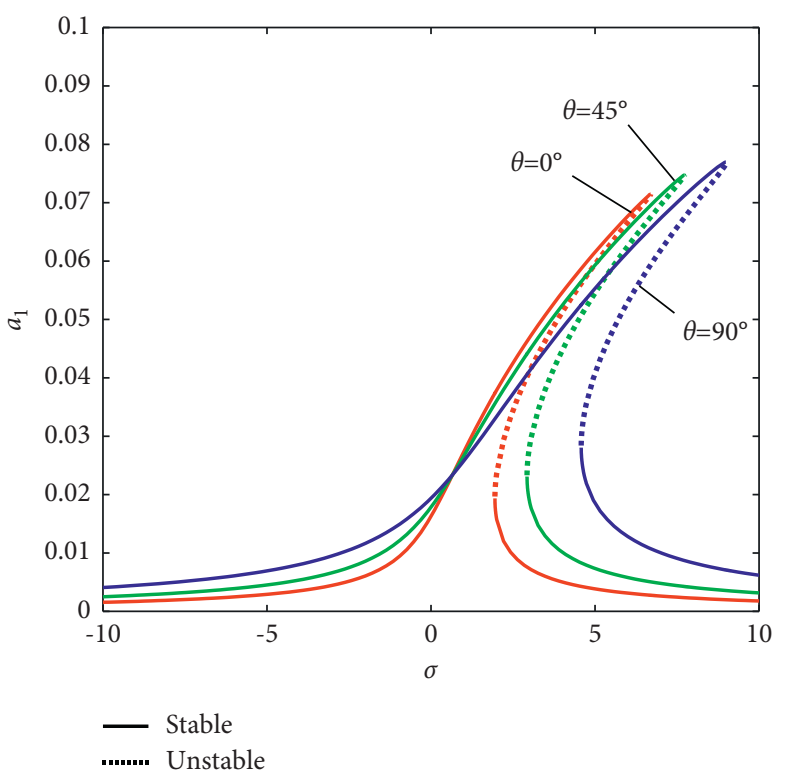

Figure 8: The effect of ply angle on frequency response curve $(\mathrm{L} / \mathrm{d}=10, \bar{c}=0.01$, case $\mathrm{I})$.

one stable branch. For large values of $\bar{c}$ and large values of $L / d$, curves are always single-valued.

Figures 15-19 show the amplitude versus ply angle with different ratios of length to diameter, detuning parameter values, cutting force coefficients, damping coefficients, and rotating speeds, respectively $\left(\bar{\Omega}=\omega_{1}\right)$. Similar to the cases in Figure $10 \sim 14$, again, for some values of $L / d, \sigma, K_{\mathrm{f}}$, or $\bar{c}$, multivalued curves can be observed.

Figures 20-24 show the amplitude versus cutting force coefficient with different ratios of length to diameter, detuning parameter values, ply angle, damping coefficients,

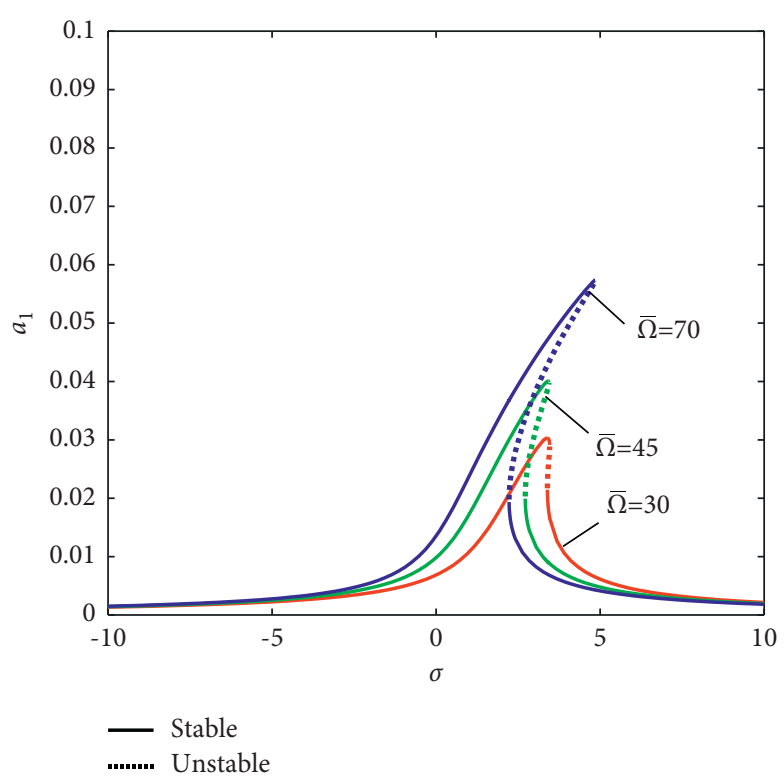

FIGURE 9: The effect of rotating speed on frequency response curve $\left(\mathrm{L} / \mathrm{d}=10, \bar{c}=0.01, \theta=0^{\circ}\right.$, case I).

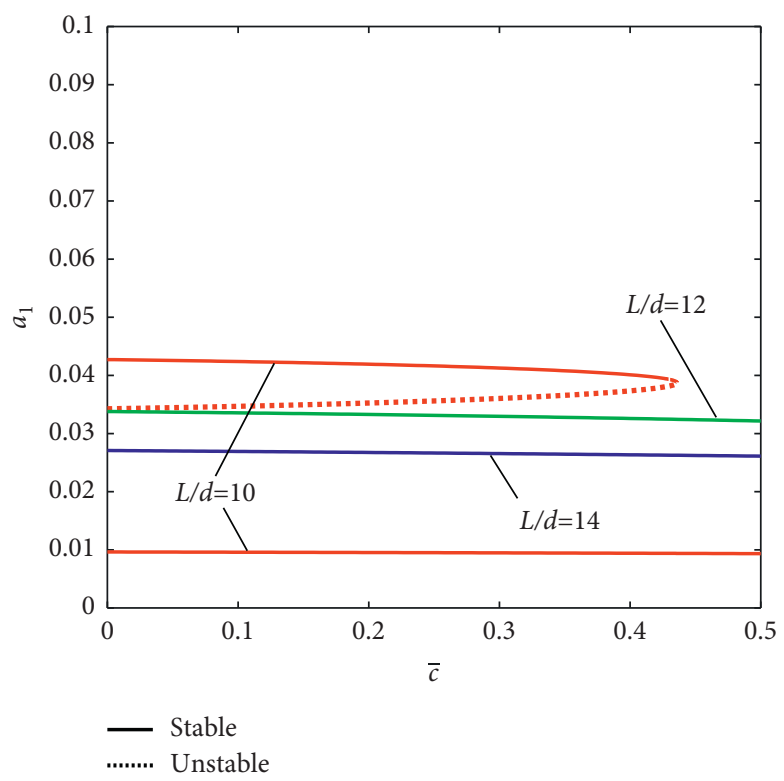

FIgURE 10: Amplitude versus damping coefficient with different ratios of length to diameter $\left(\sigma=2, \bar{c}=0.01, \theta=0^{\circ}\right.$, case I).

and rotating speeds, respectively. Again, when cutting force coefficient is less than the specified value, multivalued curves can be obtained for some values of $L / d, \sigma, \theta, \bar{c}$, or $\bar{\Omega}$.

Figures 25-27 show the effects of ply sequences (see Table 3 ) on the frequency response curve, the amplitude versus dumping coefficient, and the amplitude versus cutting force coefficient, respectively. It can be seen from Figure 25 that the configuration A leads to the largest vibration amplitude, while the configuration $\mathrm{C}$ leads to the smallest vibration amplitude. This phenomenon can also be found in Figure 8 where the vibration amplitude increases with the 


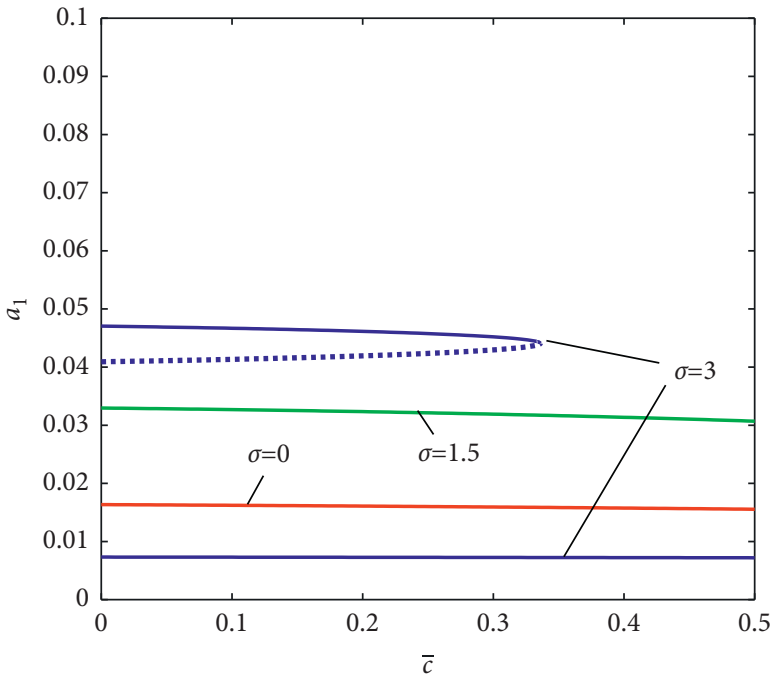

- Stable

FIgUre 11: Amplitude versus damping coefficient with different detuning parameter values $\left(\mathrm{L} / \mathrm{d}=10, \bar{c}=0.01, \theta=0^{\circ}\right.$, case I).

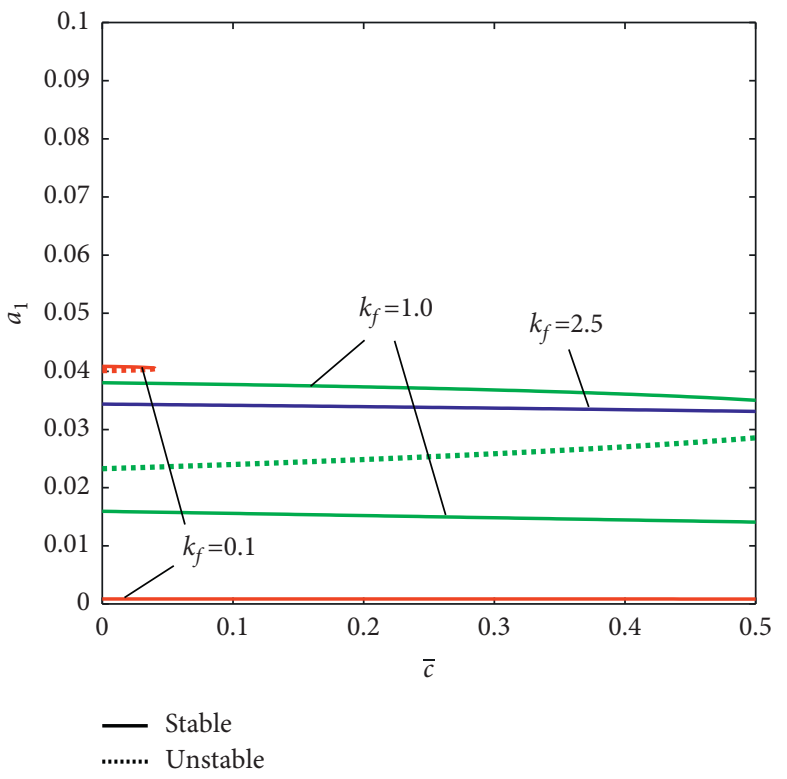

FIGURE 12: Amplitude versus damping coefficient with different cutting force coefficients $\left(\mathrm{L} / \mathrm{d}=10, \sigma=2, \theta=0^{\circ}\right.$, case I).

ply angle when using the same configuration. Again, it can be seen from Figures 26 and 27 that multivalued curves can be observed for some values of $L / d, \sigma, K_{\mathrm{f}}$, or $\bar{c}$.

3.2.2. Case II. Figures $28-33$ show the effects of ratio of length to diameter, structural damping, cutting force, ply angle, ply sequence, and rotating speed on frequency response curve, respectively, for the case $\bar{\Omega}=\omega_{2}$. As is observed, the frequency responses and effect of various parameters for the cutting system are similar to those in

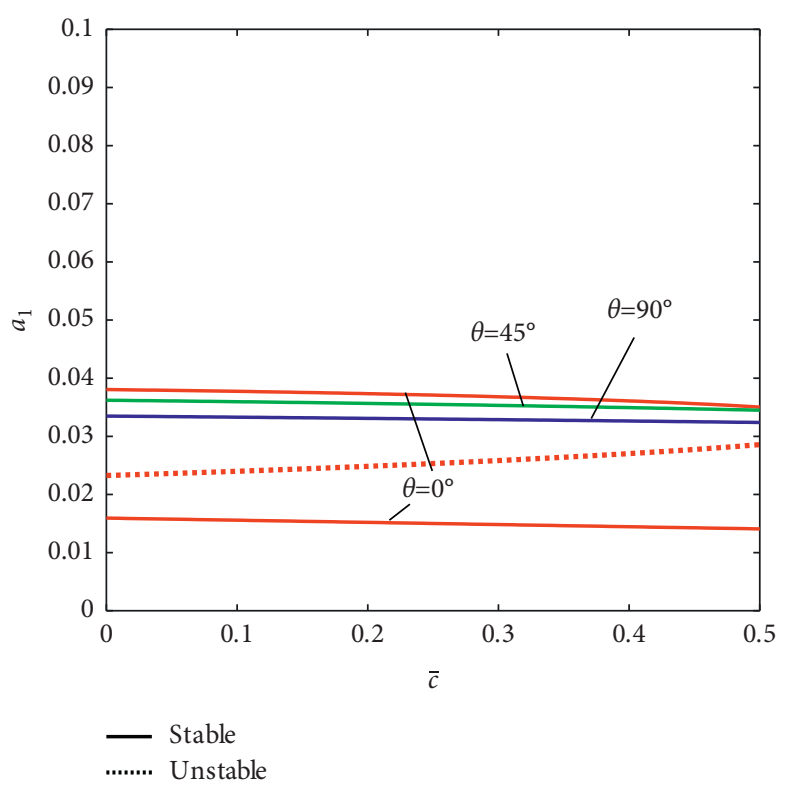

FIgURE 13: Amplitude versus damping coefficient with different ply angles $(\mathrm{L} / \mathrm{d}=10, \sigma=2$, case $\mathrm{I})$.

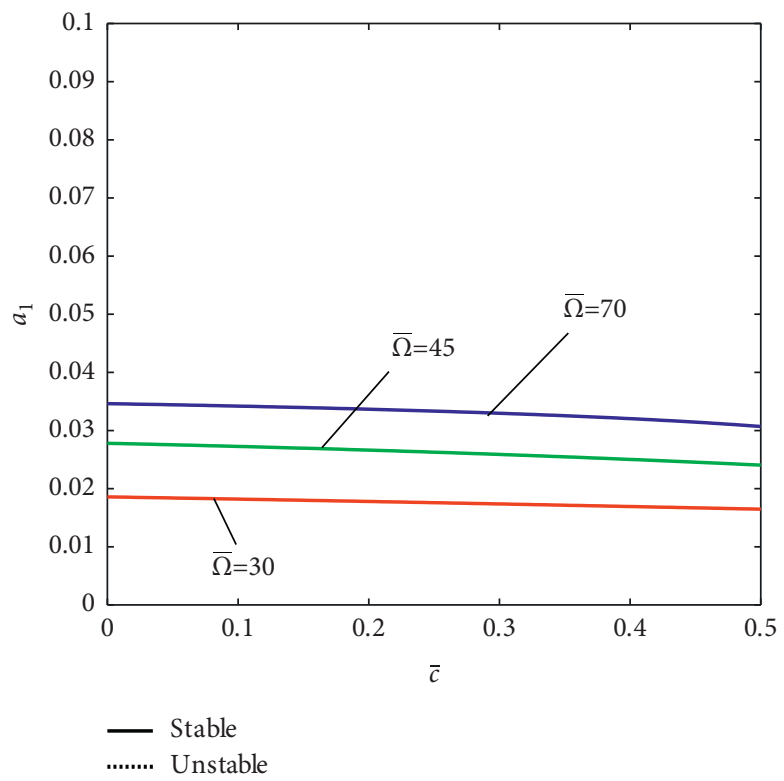

FIgURe 14: Amplitude versus damping coefficient with different rotating speed $(\mathrm{L} / \mathrm{d}=10, \sigma=2$, case $\mathrm{I})$.

case I; as ratio of length to diameter and structural damping increase, vibration amplitudes decrease. Furthermore, as cutting force coefficient, ply angle, and rotating speed increase, vibration amplitudes increase. However, for this case, vibration amplitudes are less than those of case I.

It should be noted that the equivalent damping of the cutting system is composed of the structural damping and the damping from regenerative chatter mechanism. The equivalent damping coefficient of case I can be expressed as 


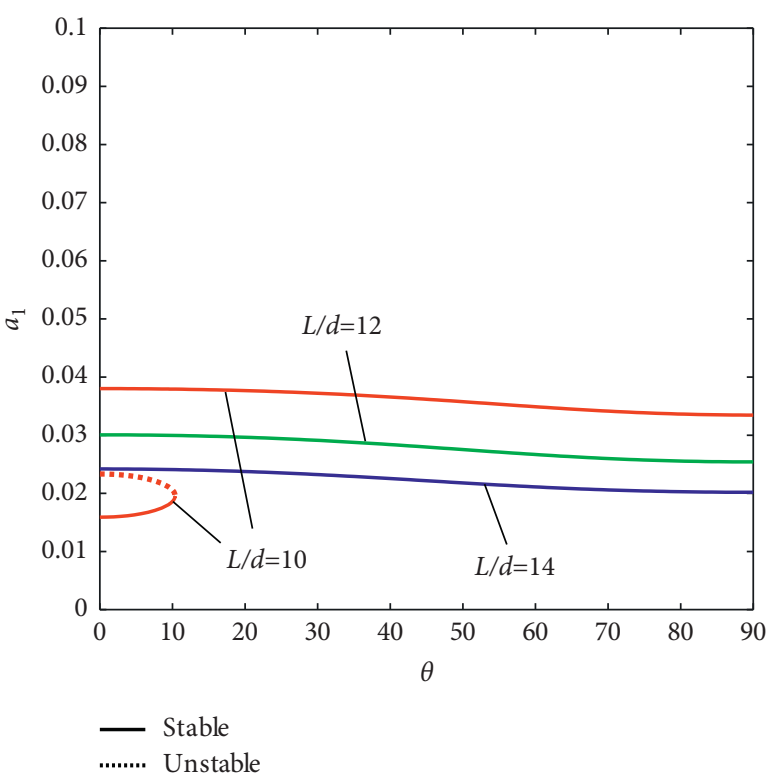

FIGURE 15: Amplitude versus ply angle with different ratios of length to diameter $(\bar{c}=0.01, \sigma=-1.5$, case I).

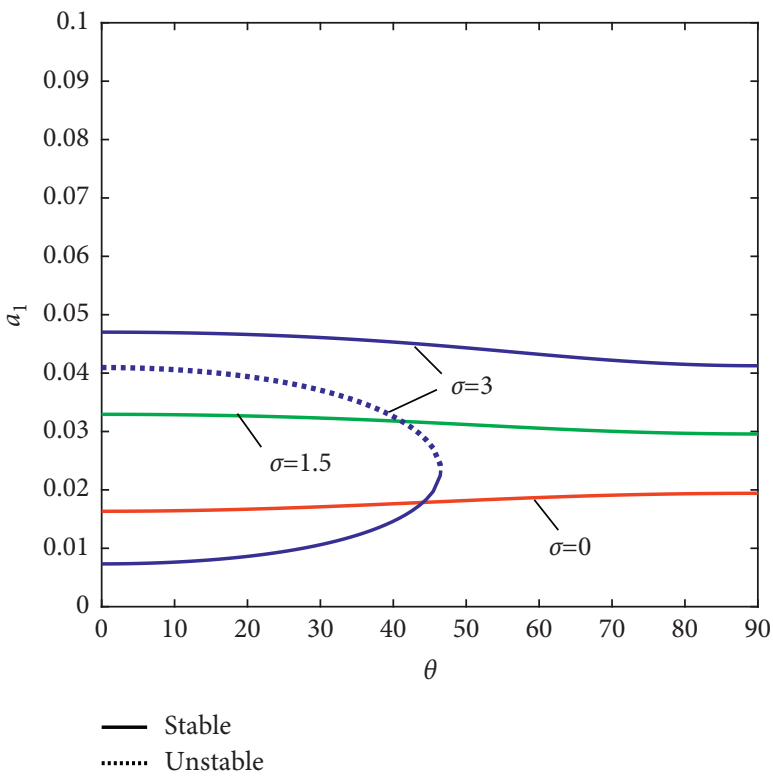

Figure 16: Amplitude versus ply angle with different detuning parameter values $(\mathrm{L} / \mathrm{d}=10, \bar{c}=0.01$, case $\mathrm{I})$.

$$
\begin{aligned}
c_{\text {equiv } 1}= & \bar{c}-\phi_{1}^{2}(1) \frac{N}{4 \pi}\left[\left(\bar{\alpha}_{0}^{\prime}+\bar{\beta}_{0}\right)\left(1-\cos \omega_{1} \bar{\tau}\right)\right. \\
& \left.+\left(\bar{\beta}_{0}^{\prime}-\bar{\alpha}_{0}\right) \sin \omega_{1} \bar{\tau}\right] /\left[\omega_{1} \int_{0}^{1} \phi_{1}^{2}(x) d x\right] .
\end{aligned}
$$

In addition, the equivalent damping coefficient of case II can be expressed as

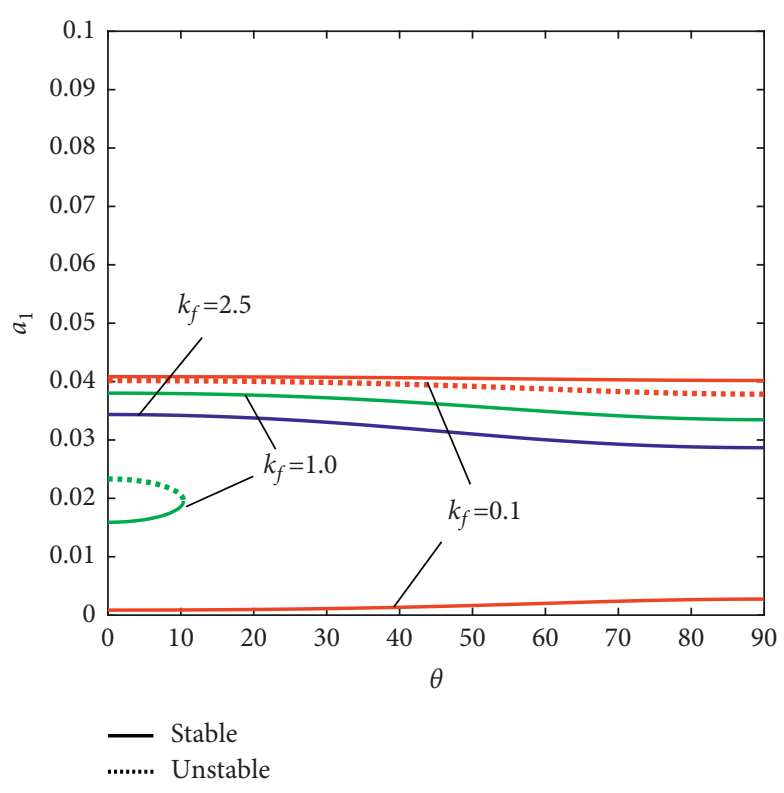

FIgURE 17: Amplitude versus ply angle with different cutting force coefficients $(\mathrm{L} / \mathrm{d}=10, \bar{c}=0.01, \sigma=2$, case I).

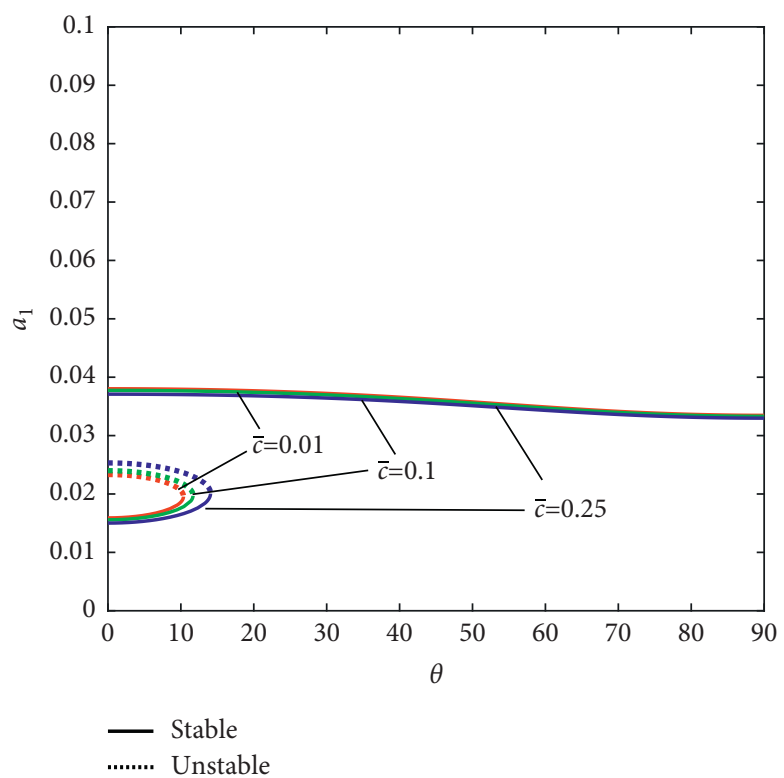

FIgURe 18: Amplitude versus ply angle with different damping coefficients $(\mathrm{L} / \mathrm{d}=10, \sigma=2$, case I).

$$
\begin{aligned}
c_{\text {equiv } 2}= & \bar{c}+\phi_{1}^{2}(1) \frac{N}{4 \pi}\left[\left(\bar{\alpha}_{0}^{\prime}+\bar{\beta}_{0}\right)\left(1-\cos \omega_{2} \bar{\tau}\right)\right. \\
& \left.+\left(\bar{\alpha}_{0}-\bar{\beta}_{0}^{\prime}\right) \sin \omega_{2} \bar{\tau}\right] /\left[\omega_{2} \int_{0}^{1} \phi_{1}^{2}(x) d x\right] .
\end{aligned}
$$

Figures 34-38 show the effect of different parameters on the equivalent damping coefficients. As is observed, the 


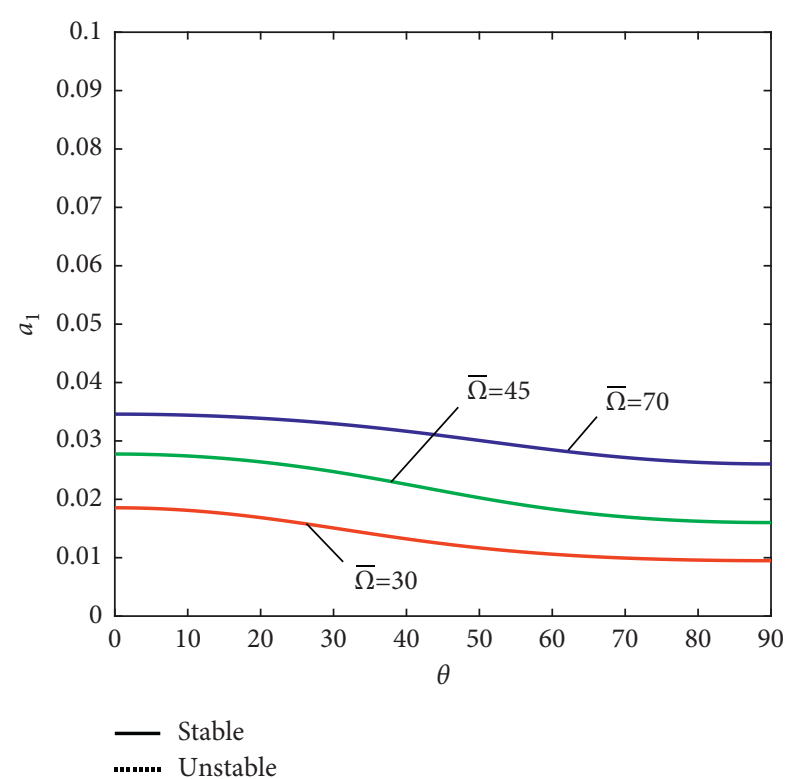

Figure 19: Amplitude versus ply angle with different rotating speeds $(\mathrm{L} / \mathrm{d}=10, \bar{c}=0.01, \sigma=2$, case $\mathrm{I})$.

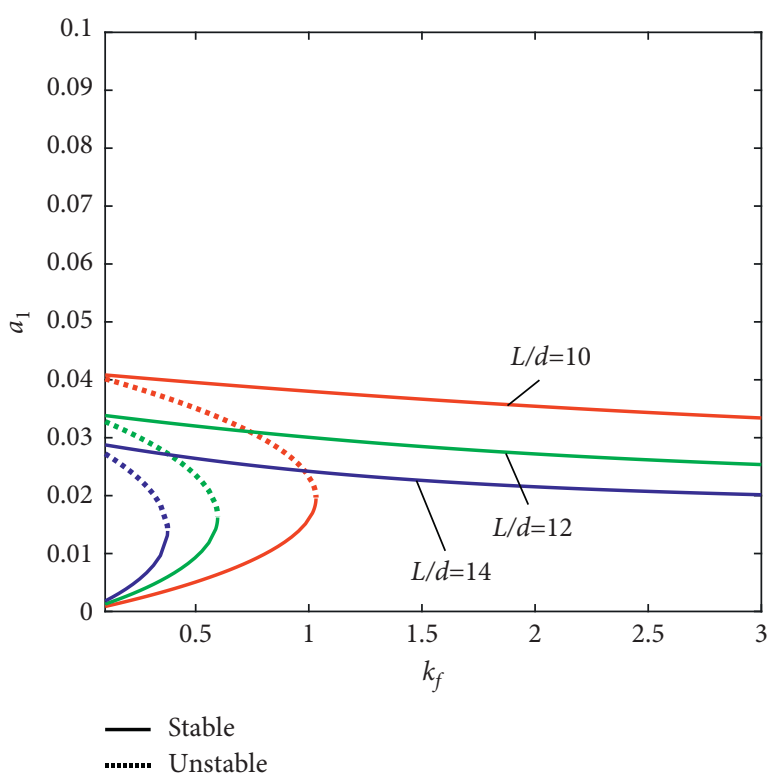

FIGURE 20: Amplitude versus cutting force coefficient with different ratios of length to diameter $\left(\theta=0^{\circ}, \bar{c}=0.01, \sigma=2\right.$, case I).

equivalent damping coefficients of case I are always larger than those of case II. This explains the reason why vibration amplitudes of case I are larger than those of case II. It can be also seen that the equivalent damping coefficients increase with ratio of length to diameter, ply angle, cutting force, and

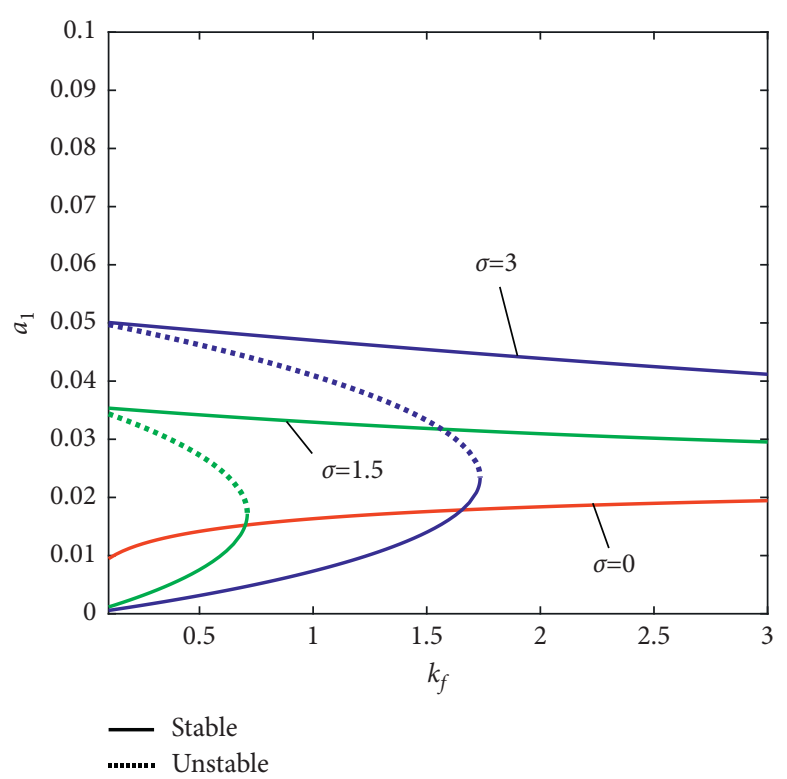

FIgURe 21: Amplitude versus cutting force coefficient with different detuning parameter values $\left(\theta=0^{\circ}, \bar{c}=0.01, \mathrm{~L} / \mathrm{d}=10\right.$, case I).

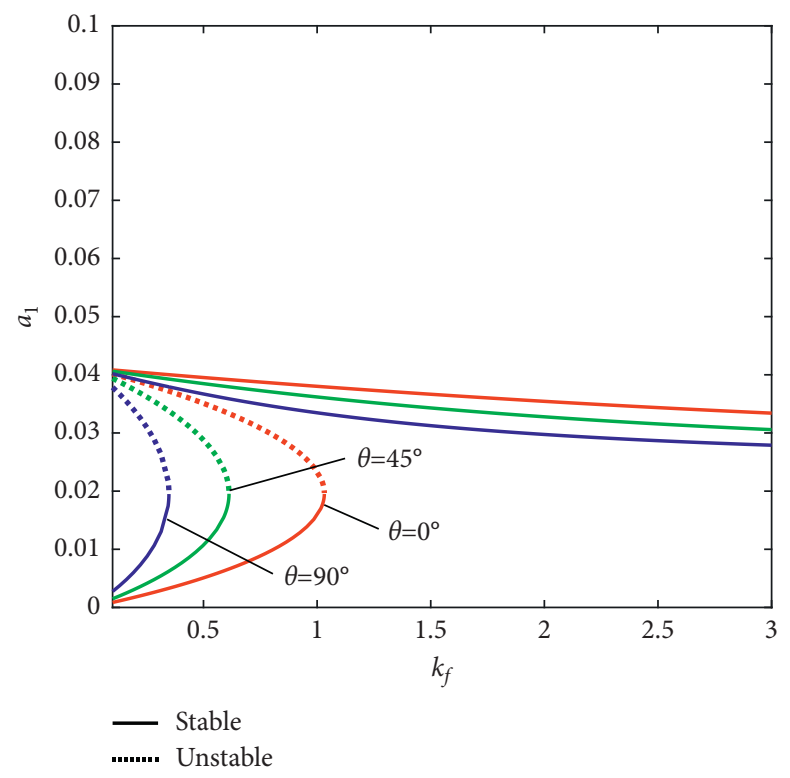

FIgUre 22: Amplitude versus cutting force coefficient with different ply angles $(\sigma=2, \bar{c}=0.01, \mathrm{~L} / \mathrm{d}=10$, case $\mathrm{I})$.

structural damping but decrease with rotating speed. Therefore, the increase of rotating speed leads to large vibration amplitudes, as shown in Figures 9 and 32. When rotating speed approaches infinity, the equivalent damping coefficient approaches the structural damping $\bar{c}$ (see Figure 38 ). 


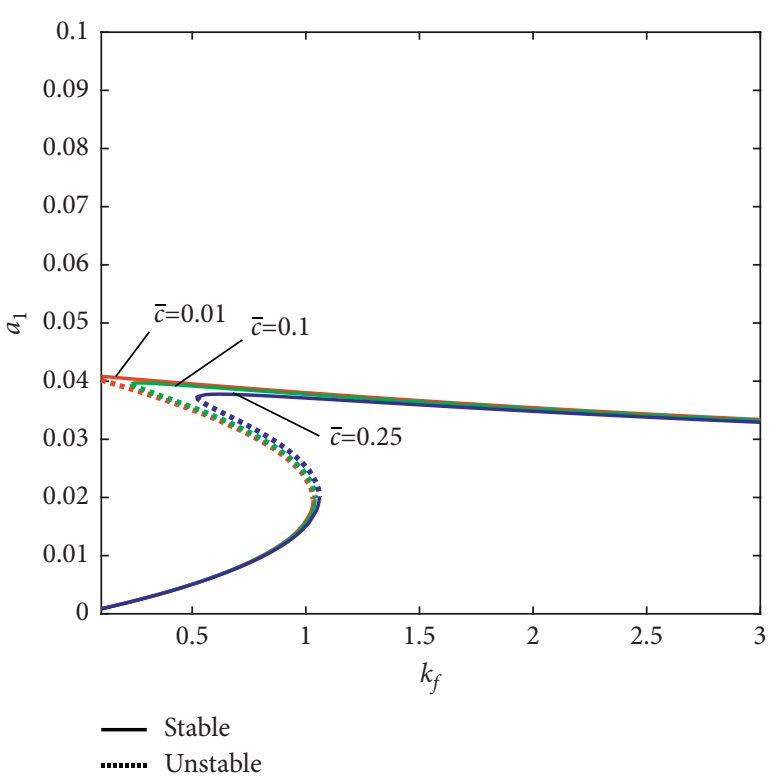

Figure 23: Amplitude versus cutting force coefficient with different damping coefficients $\left(\sigma=2, \theta=0^{\circ}, \mathrm{L} / \mathrm{d}=10\right.$, case I).

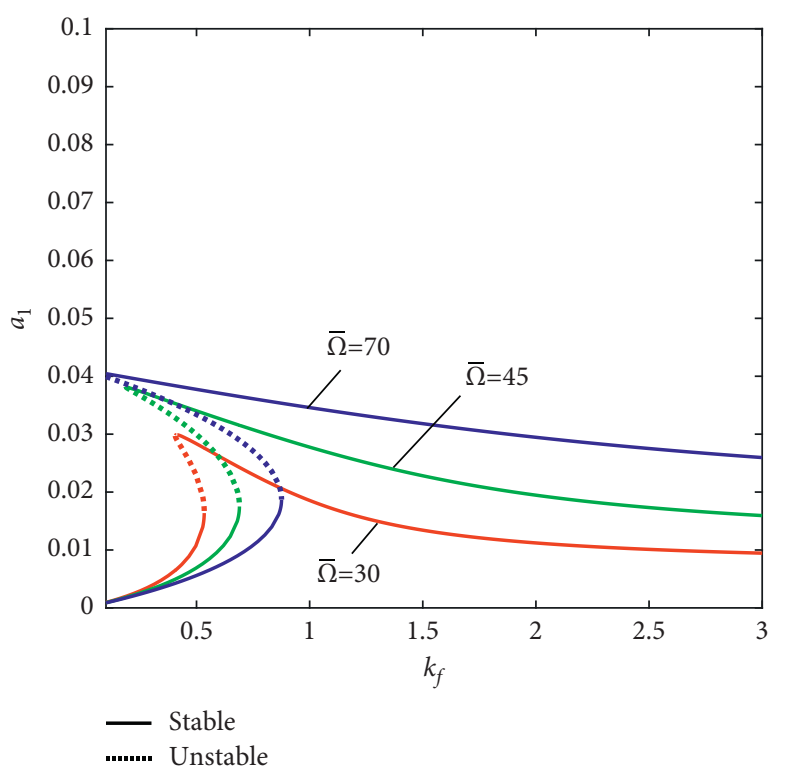

FIgUre 24: Amplitude versus cutting force coefficient with different rotating speeds $\left(\sigma=2, \bar{c}=0.01, \theta=0^{\circ}, \mathrm{L} / \mathrm{d}=10\right.$, case I).

Moreover, for some values of $L / d, \sigma, K_{\mathrm{f}}, \bar{c}, \theta$, or $\bar{\Omega}$, multivalued solutions can be found from the amplitude versus damping coefficient with different ratios of length to diameter, detuning parameter values, cutting force coefficients, ply angles, and rotating speeds, respectively (not shown).

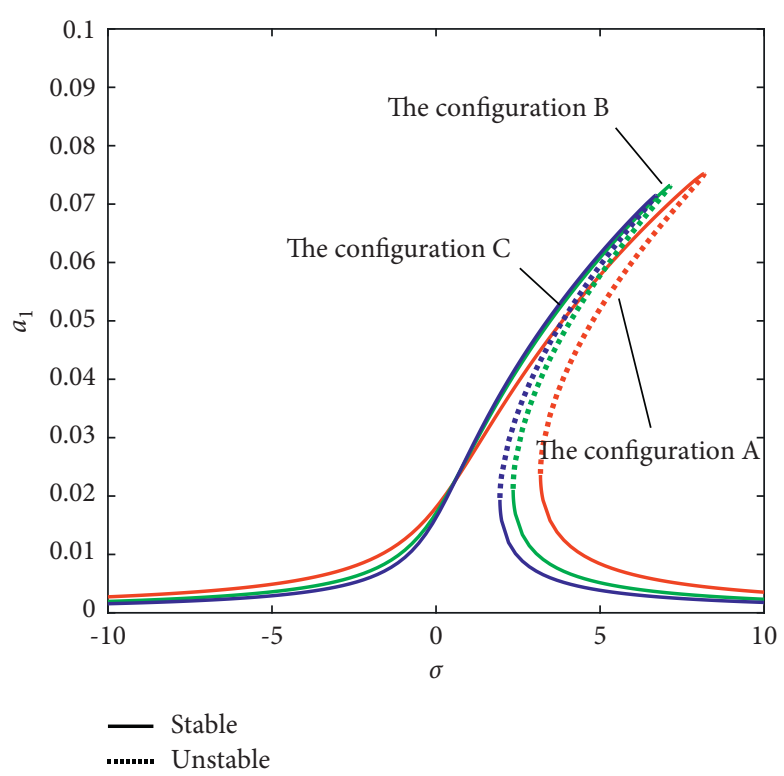

Figure 25: The frequency response curve with different ply sequences $(\bar{c}=0.05, \mathrm{~L} / \mathrm{d}=10$, case $\mathrm{I})$.

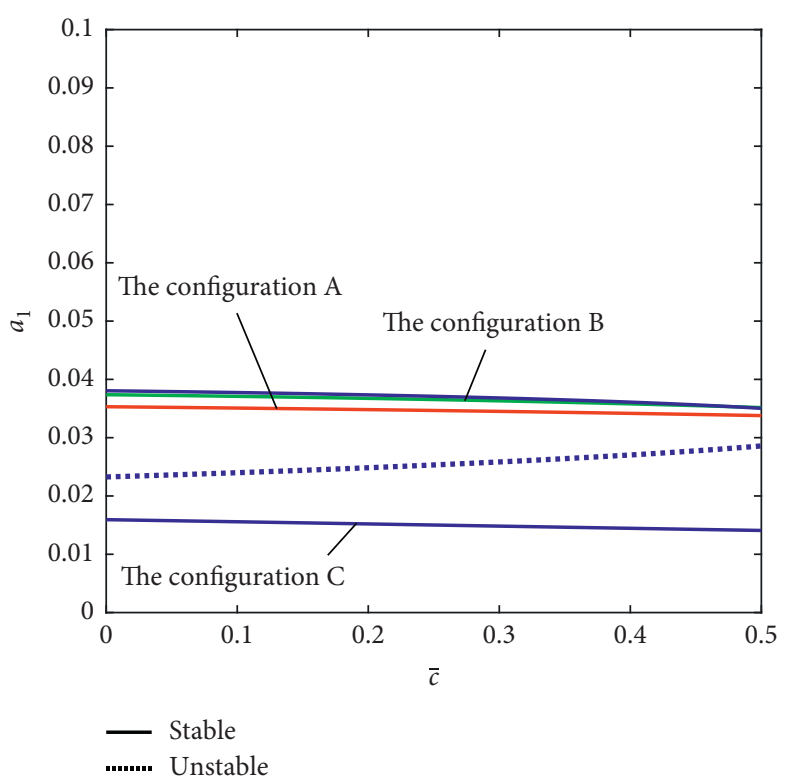

FIgUre 26: Amplitude versus dumping coefficient with different ply sequences $(\sigma=2, \mathrm{~L} / \mathrm{d}=10$, case $\mathrm{I})$.

TABle 3: Configuration of composite.

\begin{tabular}{lc}
\hline Configuration & Sequence \\
\hline A & {$\left[90^{\circ} / 90^{\circ} / 0^{\circ}\right]_{\mathrm{s}}$} \\
B & {$\left[0^{\circ} / 90^{\circ} / 0^{\circ} / 0^{\circ} / 90^{\circ} / 0^{\circ}\right]$} \\
C & {$\left[0^{\circ}\right]_{6}$} \\
\hline
\end{tabular}




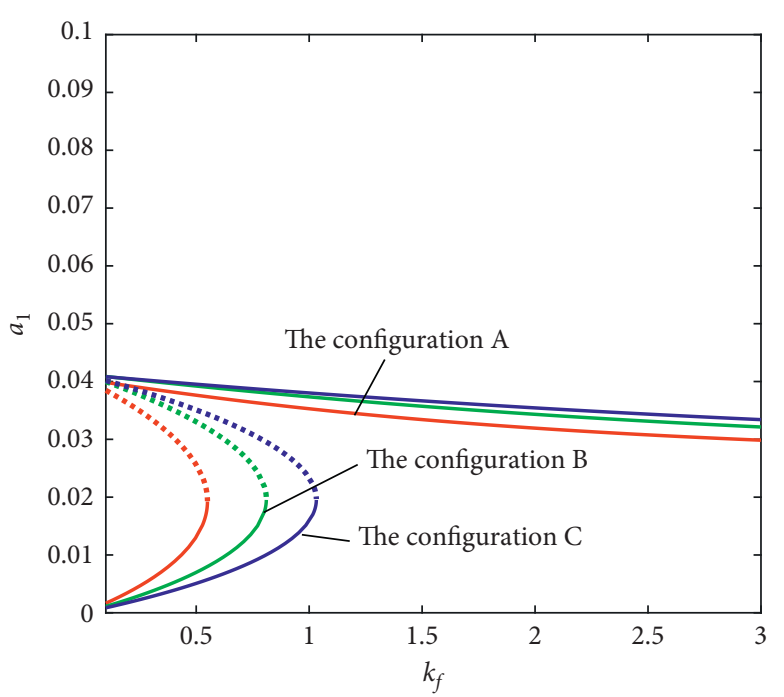

- Stable

....... Unstable

FIGURE 27: Amplitude versus cutting force coefficient with different ply sequences $(\bar{c}=0.01, \sigma=2, \mathrm{~L} / \mathrm{d}=10$, case $\mathrm{I})$.

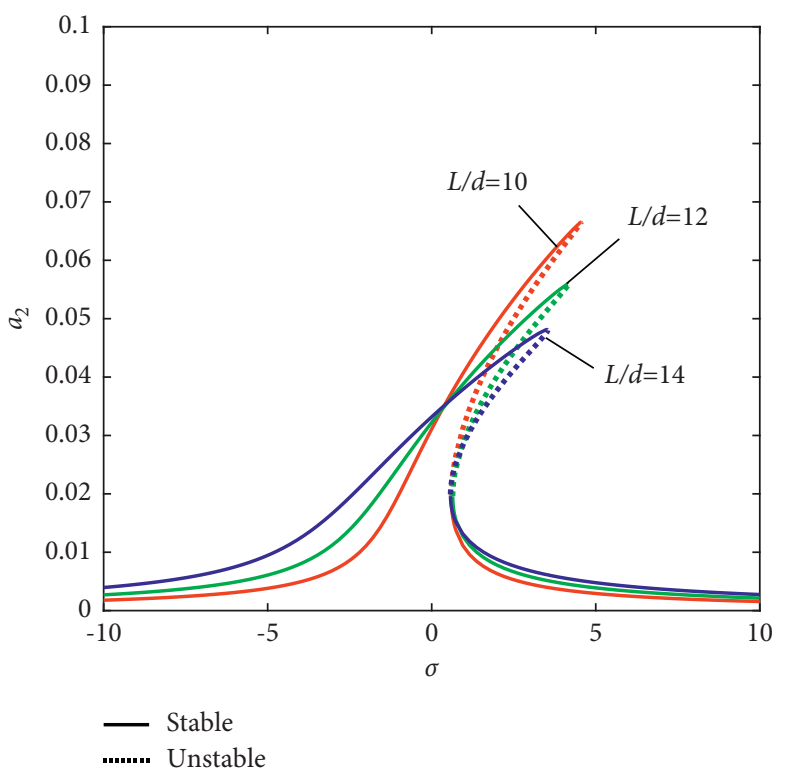

Figure 28: The effect of ratio of length to diameter on frequency response curve $\left(\theta=0^{\circ}, \bar{c}=0.01\right.$, case II).

Figure 39 shows the frequency response curve for the four cases. As is observed, the curves of cases I and III are very similar, except that the amplitude in case I is larger than that in case III. Likewise, the curves of cases II and IV are very similar, except that the amplitude in case II is larger than that in case IV.

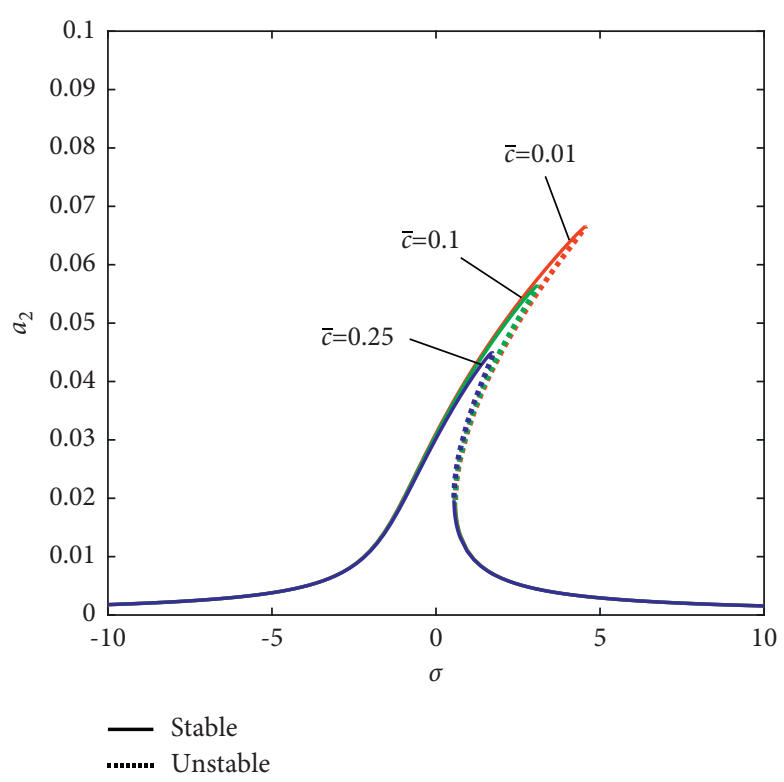

FIGURE 29: The effect of structural damping on frequency response curve $\left(\mathrm{L} / \mathrm{d}=10, \theta=0^{\circ}\right.$, case II).

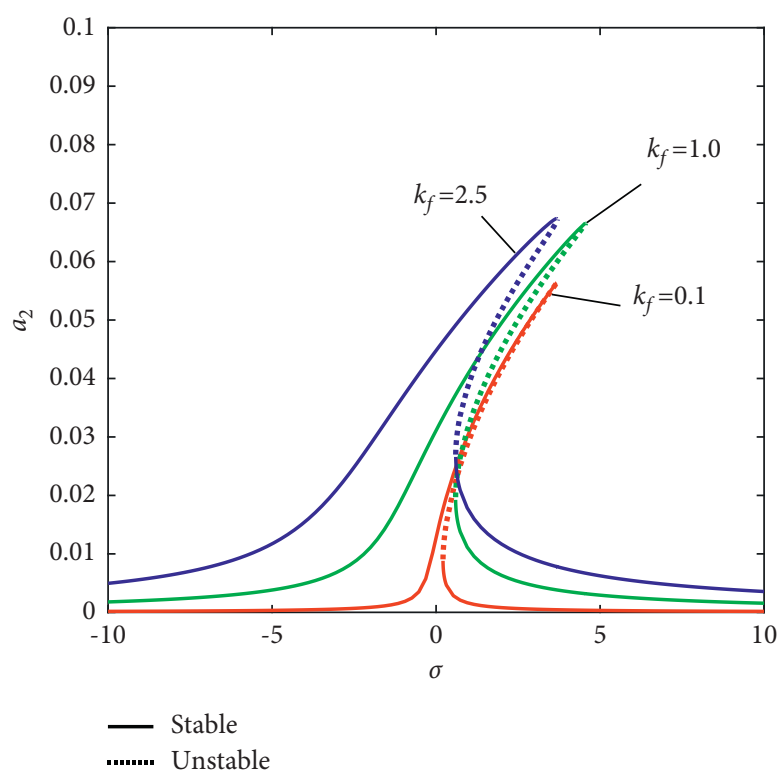

FIgUre 30: The effect of cutting force coefficient on frequency response curve $\left(\mathrm{L} / \mathrm{d}=10, \theta=0^{\circ}, \bar{c}=0.01\right.$, case II).

3.2.3. Case III. For this case, the equivalent coefficient is identical with that in case I, but the amplitude of excitation force $\left(c_{f_{\zeta_{1}}}^{2}+c_{f \eta_{1}}^{2}\right) / 4$ in this case is lower than $\left(\bar{\varsigma}_{2}^{2}+\bar{\eta}_{2}^{2}\right)$ in case I (e.g., using the same parameters $\mathrm{L} / \mathrm{d}=10, \theta=90^{\circ}$, and $K_{\mathrm{f}}=3$, the amplitudes of excitation force are 0.2695 and 0.2532 for cases I and III, respectively). Therefore, generally, 


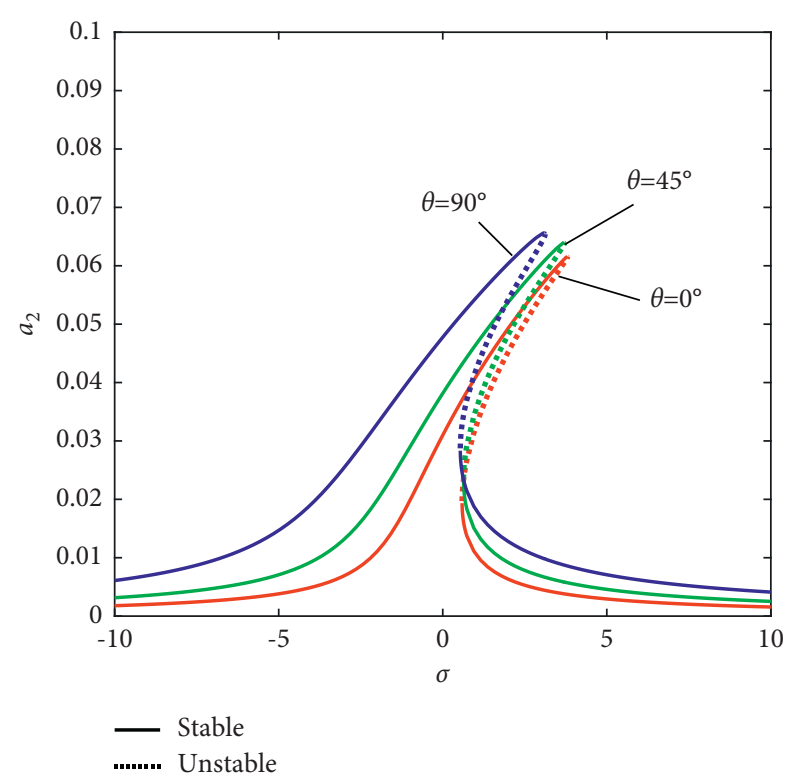

Figure 31: The effect of ply angle on frequency response curve $(\mathrm{L} / \mathrm{d}=10, \bar{c}=0.01$, case II).

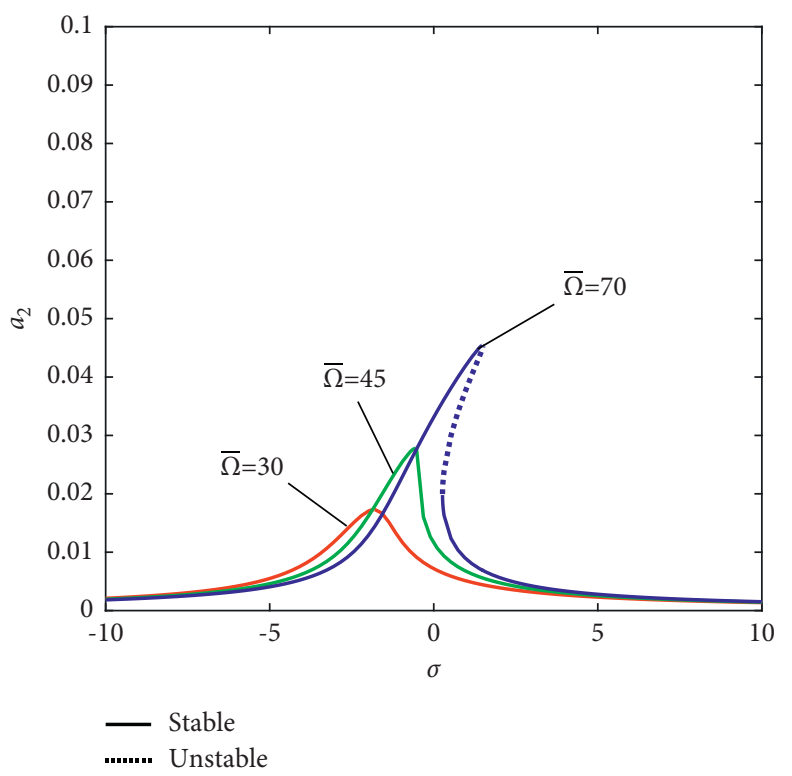

FIGURE 32: The effect of rotating speed on frequency response curve $(\mathrm{L} / \mathrm{d}=10, \bar{c}=0.01$, case II).

the behavior of nonlinear forced vibration in this case (see Figure 39) is similar to case I, despite having less vibration amplitudes compared with case I.

3.2.4. Case IV. For this case, the equivalent coefficient is identical with that in case II, and the amplitude of excitation force in this case is lower than that in in case II. Thus, under the same conditions, vibration amplitudes are generally

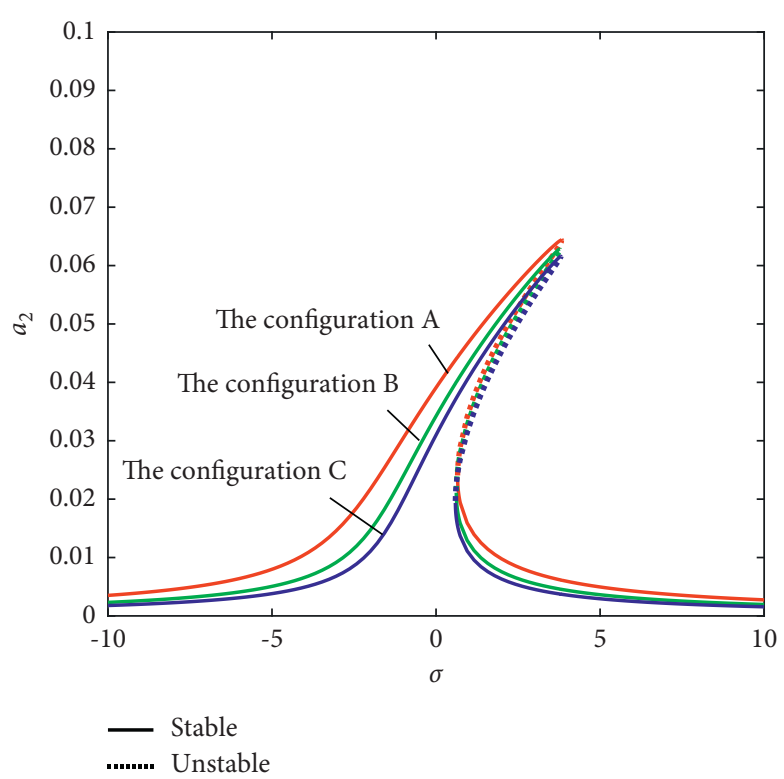

FIGURE 33: Frequency response curve with different ply configurations $(\bar{c}=0.05, \mathrm{~L} / \mathrm{d}=10$, case $\mathrm{II})$.

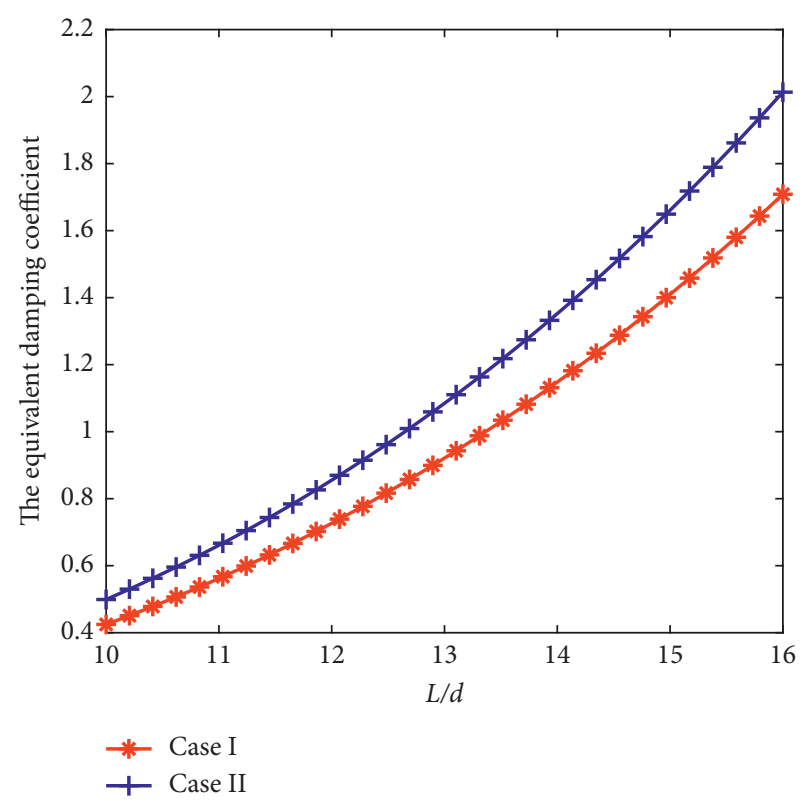

FIgURE 34: The equivalent damping coefficients versus ratio of length to diameter for cases I and II.

lower than those in case II. The results of case IV are not shown to save space.

In order to validate the calculated approximate solution using multiscale method, the numerical simulation results according to (17) are also displayed in Figures 40 and 41. These two calculated results using different methods are in good consistency. 


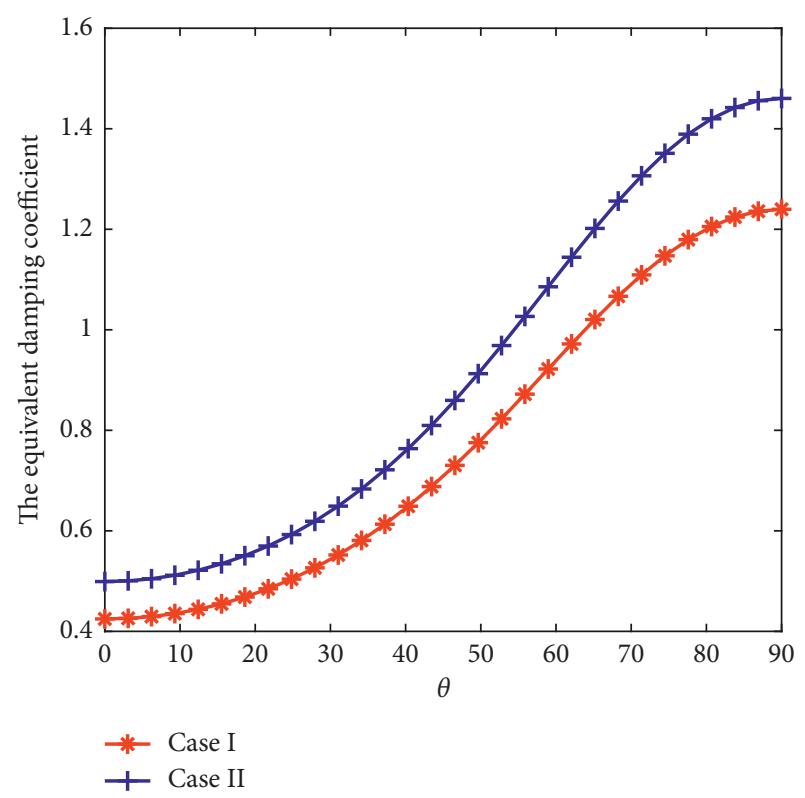

FIGURE 35: The equivalent damping coefficients versus ply angle for cases I and II.

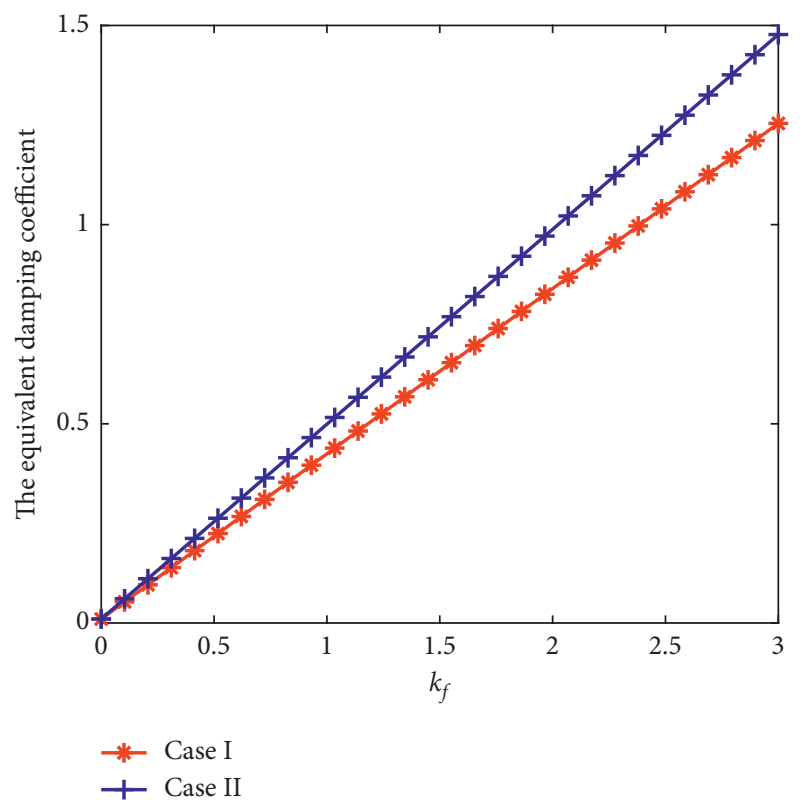

FIGURE 36: The equivalent damping coefficients versus cutting force for cases I and II.

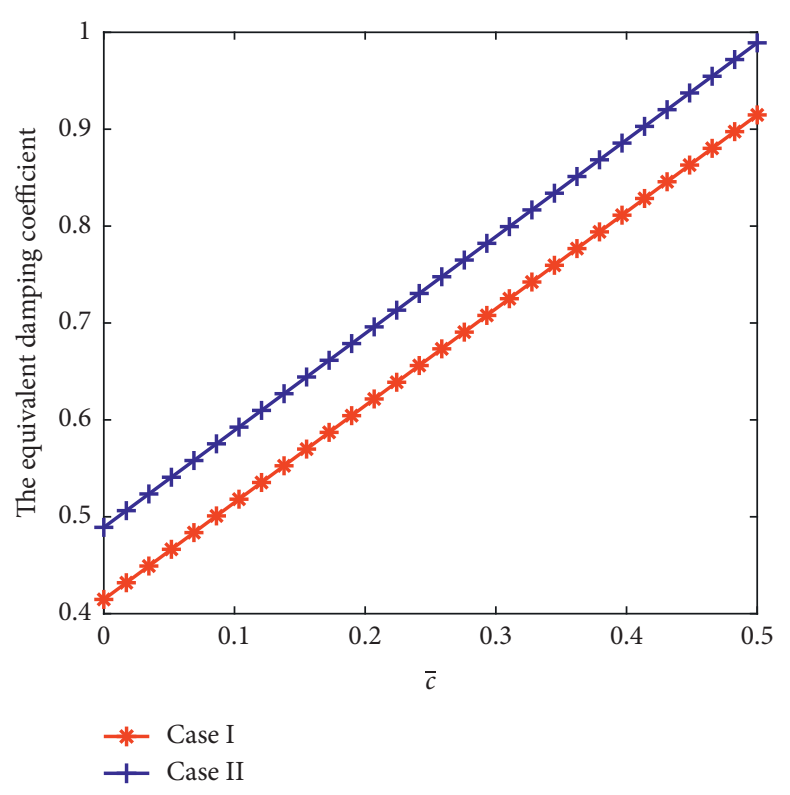

FIgURE 37: The equivalent damping coefficients versus structural damping for cases I and II.

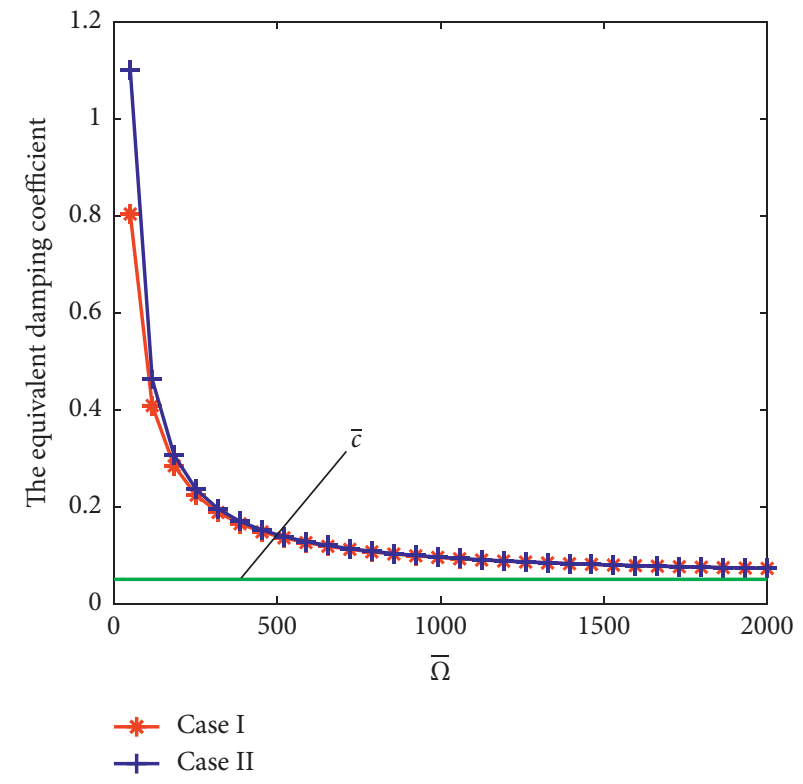

FIgURE 38: The equivalent damping coefficients versus rotating speed for cases I and II. 


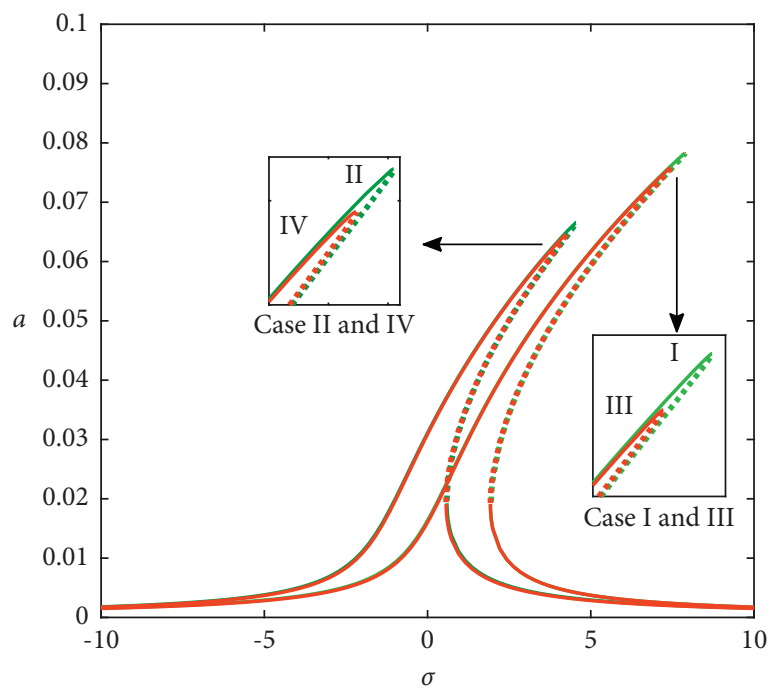

\section{- Stable}

........ Unstable

Figure 39: The frequency response curve for the four cases (L/d $=10, \bar{c}=0.01, \theta=0^{\circ}, \bar{\Omega}=100$ ).

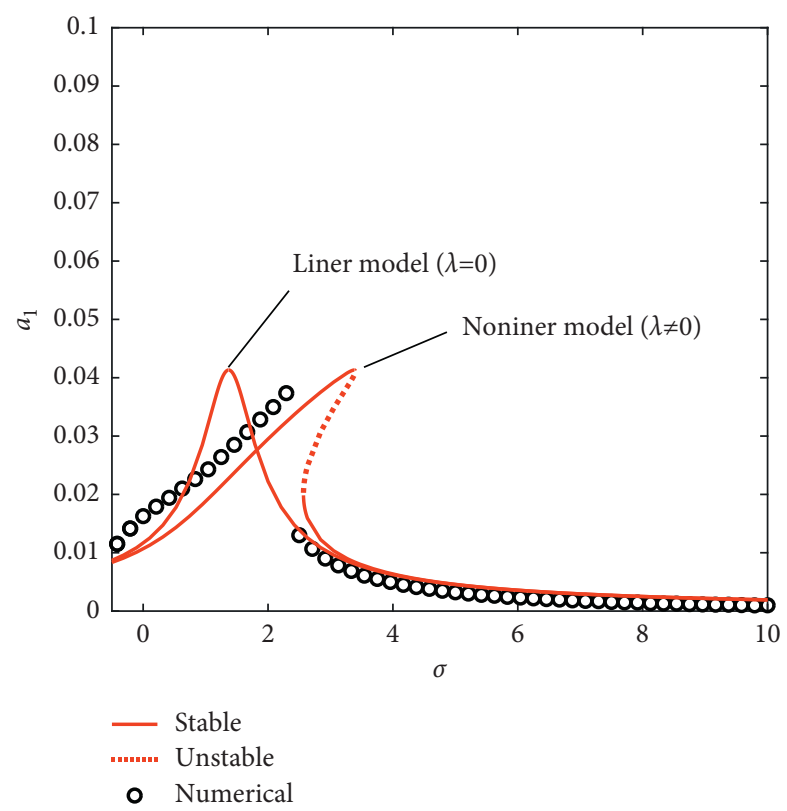

FIgURE 40: Comparison of results between the method of multiple scales and the numerical simulation $\left(\right.$ case $\mathrm{I}, \mathrm{L} / \mathrm{d}=10, \bar{c}=0.03, \theta=0^{\circ}$, $\left.\bar{\Omega}=50, K_{f}=1\right)$. 


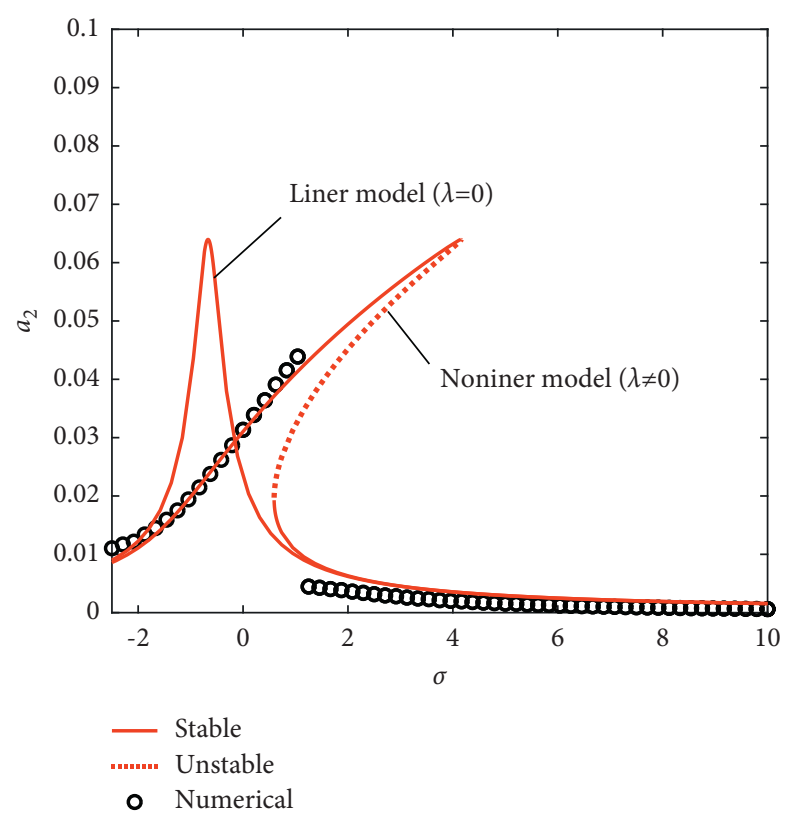

FiguRE 41: Comparison of results between the method of multiple scales and the numerical simulation (case II, L/d $=10, \bar{c}=0.03, \theta=0^{\circ}, \bar{\Omega}$ $\left.=100, K_{f}=1\right)$.

\section{Conclusions}

In this paper, a nonlinear dynamic model of the milling process with composite rotational cutting tool is presented. The cutting tool is simplified to a nonplanar bending Euler-Bernoulli beam, and the structural nonlinearity is attributed to the higher-order bending deformation of the cutting tool. A linear cutting force model considering regenerative mechanism, which includes time-delay terms and the first and second harmonic terms, is used. In addition, structural damping and gyroscopic effect are also considered. Nonlinear ordinary differential equations in the generalized coordinates are derived using the Hamilton principle and the Galerkin method. The lobe diagram of the cutting system is obtained. The multiscale method is used to construct the analytical approximate solutions of the forced vibration frequency response. It is found that, for all cases of primary resonance and superharmonic resonance, excitation of the forward (backward) mode does not produce the vibration responses in the backward (forward) mode because of not taking into account internal resonance in the proposed model. For primary and superharmonic resonance conditions, the effective nonlinearity of the cutting system with higher-order bending deformation rotating composite cutting tool is of a hard type. Jump phenomenon and multivalued solutions can be observed.

The influence of higher-order bending deformation causes the frequency response curve to bend more strongly toward right when ratio of length to diameter increases. The study also finds that the vibration amplitudes of the cutting tool increase with cutting force or ply angle. It can be seen that the damping of the cutting system has a significant influence on the vibration amplitude of the composite cutting tool. The damping capacities of the cutting system can be measured by the two different equivalent damping coefficients $c_{\text {equiv1 }}$ and $c_{\text {equiv2 }}$ which include the structural damping and the damping from regenerative chatter mechanism. Cases I and III have the same equivalent damping coefficient $c_{\text {equiv } 1}$, and cases II and IV have the same equivalent damping coefficient $c_{\text {equiv2 }}$. It has been found that $c_{\text {equiv2 }}$ is larger than $c_{\text {equiv1 }}$, so the vibration amplitudes of case I are larger than those of case II. In addition, for all resonant cases, as rotating speed decreases or structural damping increases, the equivalent damping coefficient is strengthened, and consequently less vibration amplitudes are observed. Therefore, from this point of view, low rotating speeds are preferable to keep vibration amplitudes small. [38].

\section{Abbreviations}

$T:$

L: $\quad$ Length of the cutting tool

$t: \quad$ Time

$\rho: \quad$ Density

A: $\quad$ Cross-sectional area

I: $\quad$ Second moment of area of the beam cross

$v, w:$

$x, y, z$ : section

$\psi_{y}, \psi_{z}$ : $\quad$ Rotation angles of the cross section around the $y$ and $z$ axes

$\Omega$ : $\quad$ Rotating speed of the shaft

$\bar{Q}_{11}$ : Off-axis stiffness coefficient of the $k$ th layer

$U: \quad$ Potential energy

$W_{c}$ : $\quad$ Rayleigh dissipative energy function of the cutting tool

$c: \quad$ Structural damping coefficient

$\delta$ : Variational operator 


$\begin{array}{ll}\delta W: & \text { Virtual work of the cutting force } \\ F_{y,} F_{z}: & \text { Linear regenerative cutting forces } \\ \tau: & \text { Delay time of milling process } \\ N: & \text { The number of teeth } \\ K_{\mathrm{tc}}: & \text { Cutting force coefficients in the tangential } \\ & \text { directions } \\ K_{\mathrm{te}}: & \text { Edge force coefficients in the tangential } \\ K_{\mathrm{rc}}: & \text { directions } \\ K_{\mathrm{re}}: & \text { Cutting force coefficients in the radial } \\ & \text { directions } \\ a: & \text { Edge force coefficients in the radial } \\ \mathrm{c}_{f}: & \text { directions } \\ (E I)_{\text {equiv }}, & \text { Axial cutting depth } \\ (E A)_{\text {equiv }}: & \text { Feed per tooth per revolution } \\ (\rho I)_{\text {equiv }}, & \text { the cutting tool } \\ (m)_{\text {equiv }}: & \text { Equivalent diametrical mass moment of } \\ \rho_{k}: & \text { inertia and equivalent mass per unit length } \\ r_{\mathrm{k}}, r_{\mathrm{k}+1}: & \text { Density of the } k \text { th layer } \\ \phi_{1}(\bar{x}): & \text { Inner diameter and outer diameter of the } k \text { th } \\ \omega_{1}, \omega_{2}: & \text { layer } \\ \varepsilon: & \text { Mode function } \\ i: & \text { Forward and backward whirling frequencies }\end{array}$

\section{Appendix}

\section{A. The Stain Energy of the Composite Cutting Tool}

If the torsional deformation is negligible, the bending displacements of the composite cutting tool in $x, y$, and $z$ directions are as follows:

$$
u_{x}=-z \psi_{y}+y \psi_{z}, u_{y}=v, u_{z}=w .
$$

The strain in the $x$ direction is

$$
\varepsilon_{x x}=\varepsilon_{l}+\varepsilon_{n l},
$$

where

$$
\varepsilon_{l}=-z \frac{\partial \psi_{y}}{\partial x}+y \frac{\partial \psi_{z}}{\partial x}, \varepsilon_{n l}=\frac{1}{2} \psi_{y}^{2}+\frac{1}{2} \psi_{z}^{2}
$$

where $\varepsilon_{l}$ and $\varepsilon_{n l}$ are the linear strain and the nonlinear strain generated in high-order bending deformation, respectively.

Bending energy for the composite cutting tool can be expressed as

$$
U=\frac{1}{2} \int_{0}^{L} \iint_{A}\left(\sigma_{x x} \varepsilon_{x x}\right) \mathrm{d} A \mathrm{~d} x
$$

The elastic stress-strain relations of the laminate cutting tool can be expressed as

$$
\sigma_{x x}=\bar{Q}_{11} \varepsilon_{x x} .
$$

Employing (A.2), (A.3), and (A.5), (A.4) can be rewritten as

$$
U=\frac{1}{2} \int_{0}^{L} \iint_{A} \bar{Q}_{11}\left(z^{2}\left(\frac{\partial \psi_{y}}{\partial x}\right)^{2}+y^{2}\left(\frac{\partial \psi_{z}}{\partial x}\right)^{2}-2 y z \frac{\partial \psi_{y}}{\partial x} \frac{\partial \psi_{z}}{\partial x}+\frac{1}{4} \psi_{y}^{4}+\frac{1}{4} \psi_{z}^{4}+\frac{1}{2} \psi_{y}^{2} \psi_{z}^{2}-2\left(z \frac{\partial \psi_{y}}{\partial x}+y \frac{\partial \psi_{z}}{\partial x}\right)\left(\frac{1}{2} \psi_{y}^{2}+\frac{1}{2} \psi_{z}^{2}\right)\right)^{2} d A d x
$$

Due to the symmetry of the cross section, the third and seventh terms in the above equation are equal to zero.
Therefore, the strain energy of the composite cutting tool can be reduced as follows:

$$
U=\frac{(E I)_{\text {equiv }}}{2} \int_{0}^{L}\left(\left(\frac{\partial \psi_{y}}{\partial x}\right)^{2}+\left(\frac{\partial \psi_{z}}{\partial x}\right)^{2}\right) d x+\frac{(E A)_{\text {equiv }}}{2} \int_{0}^{L}\left(\frac{1}{4} \psi_{y}^{4}+\frac{1}{4} \psi_{z}^{4}+\frac{1}{2} \psi_{y}^{2} \psi_{z}^{2}\right) d x
$$

\section{B. The Expression of the Off-Axis Stiffness Coefficient}

The off-axis stiffness coefficient of the $k$ th layer for the composite cutting tool is defined as

$$
\bar{Q}_{11}=C_{11} \cos ^{4} \theta^{(k)}+C_{22} \sin ^{4} \theta^{(k)}+2\left(C_{12}+2 C_{66}\right) \sin ^{2} \theta^{(k)} \cos ^{2} \theta^{(k)},
$$

where $\theta^{(k)}$ is the layer angle of each layer of the material.

The expressions of $C_{11}, C_{12}, C_{22}$, and $C_{66}$ are as follows:

$$
C_{11}=\frac{E_{11}}{1-v_{12} v_{21}}, C_{12}=\frac{v_{12} E_{11}}{1-v_{12} v_{21}}=\frac{v_{21} E_{22}}{1-v_{12} v_{21}}, C_{22}=\frac{E_{22}}{1-v_{12} v_{21}}, C_{66}=C_{12},
$$


where

$$
v_{21}=\frac{v_{12} E_{11}}{E_{22}} \text {. }
$$

\section{Data Availability}

The data used to support the findings of this study are included within the article.

\section{Conflicts of Interest}

The authors declare that they have no conflicts of interest.

\section{Acknowledgments}

The research was funded by the https://doi.org/10.13039/ 501100001809National Natural Science Foundation of China (Grant No. 11672166).

\section{References}

[1] K. Seto and N. Tominari, "Effect of a variable stiffness-type dynamic damper on machine tools with long overhung ram," Bulletin of JSME, vol. 19, no. 137, pp. 1270-1277, 1976.

[2] D. J. P. Hartog, Mechanical Vibrations, McGraw-Hill, New York, 1956.

[3] S. Ema and E. Marui, "Suppression of chatter vibration of boring tools using impact dampers.International," Journal of Machine Tools and Manufacture, vol. 40, no. 8, pp. 1141-1156, 2008.

[4] S. Nagano, T. Koizumi, T. Fujii, N. Tsujiuchi, H. Ueda, and S. Kobe, "Development of a composite boring bar. Compos Structures," Composite Structures, vol. 38, pp. 531-539, 1997.

[5] D. G. Lee, H. Y. Hwang, and J. K. Kim, "Design and manufacture of a carbon fiber epoxy rotating boring bar," Composite Structures, vol. 60, pp. 115-124, 2003.

[6] S. Ghorbani, V. A. Rogov, A. Carluccio, and P. S. Belov, "The effect of composite boring bars on vibration in machining process," International Journal of Advanced Manufacturing Technology, vol. 105, no. 1-4, pp. 1157-1174, 2019.

[7] F. Taylor, "On the art of cutting metals," Trans. ASME, vol. 28, pp. 31-350, 1907.

[8] S. A. Tobias and W. Fishnick, "The chatter of lathe tools under orthogonal cutting conditions," Transactions of the American Society of Mechanical Engineers, vol. 80, pp. 1079-1086, 1958.

[9] H. E. Merritt, "Theory of self-excited machine tool chatter, condition to machine tool chatter research-1," Transactions of the American Society of Mechanical Engineers, Journal of Engineering for Industry, vol. 87, pp. 447-454, 1965.

[10] S. Kato and E. Marui, "On the cause of regenerative chatter due to workpiece deflection," Transactions of the American Society of Mechanical Engineers, Journal of Engineering for Industry, vol. 96, pp. 179-186, 1974.

[11] D. W. Wu and C. R. Li, "An analytical model of cutting dynamics," Transactions of the American Society of $\mathrm{Me}$ chanical Engineers, Journal of Engineering for Industry, vol. 107, pp. 107-188, 1985.

[12] Y. Altintas, Manufacturing Automation, Metal Cutting Mechanics, Machine Tool Vibrations, and CNC Design, Cambridge University Press, New York, 2000.

[13] E. Budak and Y. Altintas, "Analytical prediction of chatter stability in milling. Part I: general formulation," Trans. ASME,
Journal of Dynamic Systems, Measurement, and Control, vol. 120, pp. 22-30, 1998.

[14] E. Budak and Y. Altintas, "Analytical prediction of chatter stability in milling. Part II: application of the general formulation to common milling systems," Trans. ASME, Journal of Dynamic Systems, Measurement, and Control, vol. 120, pp. 31-36, 1998.

[15] N. H. Hanna and S. A. Tobias, "A theory of nonlinear regenerative chatter," Transactions of American Society of Mechanical Engineers, Journal of Engineering for Industry, vol. 96, pp. 247-255, 1974.

[16] L. Vela-Martinez, J. C. Jáuregui-Correa, O. M. GonzálezBrambila, and H. R. Gilberto, "Instability conditions due to structural nonlinearities in regenerative chatter," Nonlinear Dynamics, vol. 56, pp. 415-427, 2009.

[17] N. Deshpande and M. S. Fofana, "Nonlinear regenerative chatter in turning," International Journal of Computer Integrated Manufacturing, vol. 17, pp. 107-112, 2001.

[18] G. Stepan, T. Insperger, and R. Szalai, "Delay, parametric excitation, and the nonlinear dynamics of cutting process," International Journal of Bifurcation and Chaos, vol. 15, no. 9, pp. 2783-2798, 2005.

[19] J. R. Pratt, Vibration Control for Chatter Suppression, Ph.D. Thesis, Virginia Polytechnic Institute and State University, Blacksburg, VA, 1997.

[20] H. Moradi, M. R. Movahhedy, and G. Vossoughi, "Bifurcation analysis of milling process with tool wear and process damping: regenerative chatter with primary resonance," Nonlinear Dynamics, vol. 70, no. 1, pp. 481-509, 2012.

[21] H. Moradi, G. Vossoughi, M. R. Movahhedy, and T. A. Mohammad, "Forced vibration analysis of the milling process with structural nonlinearity, internal resonance, tool wear and process damping effects," International Journal of Non-linear Mechanics, vol. 54, pp. 22-34, 2013.

[22] H. Moradi, M. R. Movahhedy, and G. Vossoughi, "Dynamics of regenerative chatter and internal resonance in milling process with structural and cutting force nonlinearities," Journal of Sound and Vibration, vol. 331, pp. 3844-3865, 2012.

[23] P. N. Rao, U. R. K. Rao, and J. S. Rao, "Towards improved design of boring bars part 1: dynamic cutting force model with continuous system analysis for the boring bar," Journal of Machine Tools Manufacture, vol. 28, no. 1, pp. 33-44, 1988.

[24] L. Andren, L. Hakansson, A. Brandt, and I. Claesson, "Identification of dynamic properties of boring bar vibrations in a continuous boring operation," Journal of Mechanical Systems and Signal Processing, vol. 18, no. 4, pp. 869-901, 2004.

[25] L. Andren, L. Hakansson, A. Brandt, and I. Claesson, "Identification of motion of cutting tool vibration in a continuous boring operation-correlation to structural properties," Journal of Mechanical Systems and Signal Processing, vol. 18, pp. 903-927, 2004.

[26] B. Moetakef-Imani and N. Z. Yussefian, "Dynamic simulation of boring process," International Journal of Machine Tools and Manufacture, vol. 49, pp. 1096-1103, 2009.

[27] M. Sortino, G. N. Totis, and F. Prosperi, "Modeling the dynamic properties of conventional and high-damping boring bars," Mechanical Systems and Signal Processing, vol. 34, pp. 340-352, 2013.

[28] B. Huang, "The drilling vibration behavior of a twisted microdrill," ASME, Journal of Manufacturing Science and Engineering, vol. 126, pp. 719-726, 2004.

[29] S. Filiz and O. B. Ozdoganlar, "Micro-end mill dynamics including the actual fluted geometry and setup errors-part I: 
model development and numerical solution," ASME, Journal of Manufacturing Science and Engineering, vol. 130, no. 03, pp. 11-19, 2008.

[30] M. R. Movahhedy and P. Mosaddegh, "Prediction of chatter in high speed milling including gyroscopic effects," International Journal of Machine Tools and Manufacture, vol. 46, no. 9, pp. 996-1001, 2005.

[31] S. A. Tajalli, M. R. Movahhedy, and J. Akbari, "Chatter instability analysis of spinning micro-end mill with process damping effect via semi-discretization approach," Acta Mechanics, vol. 225, pp. 715-734, 2014.

[32] C. J. Li, A. G. Ulsoy, and W. J. Endres, "The effect of flexibletool rotation on regenerative instability in machining," Journal of Manufacturing Science and Engineering, vol. 125, no. 1, pp. 39-47, 2003.

[33] W. Kim, A. Argento, and R. A. Scott, "Forced vibration and dynamic stability of a rotating tapered composite Timoshenko shaft: bending motions in end-milling operations," Journal of Sound and Vibration, vol. 246, no. 4, pp. 583-600, 2001.

[34] M. R. Shad, G. Michon, and A. Berlioz, "Modeling and analysis of nonlinear rotordynamics due to higher order deformation in bending," Applied Mathematical Modelling, vol. 35, pp. 2145-2159, 2011.

[35] A. H. Nayfeh and P. F. Pai, Linear and Nonlinear Structural Mechanics, John Wiley \& Sons, New York, 2004.

[36] A. H. Nayfeh, Introduction to Perturbation Techniques, WileyInterscience, New York, 1981.

[37] D. A. Saravanos, D. Varelis, T. S. Plagianakos, and N. Chrysochoidis, "A shear beam finite element for the damping analysis of tubular laminated composite beams," Journal of Sound and Vibration, vol. 291, pp. 802-823, 2006.

[38] G. Tlusty, Manufacturing Process and Equipment, PrenticeHall, Englewood, 2000. 